\title{
Altered Dynamics of Canonical Feedback Inhibition Predicts Increased Burst Transmission in Chronic Epilepsy
}

\author{
Leonie Pothmann, ${ }^{1 \star}$ (C) Christian Klos, ${ }^{2 \star}$ (O) Oliver Braganza, ${ }^{1 \star}$ Sarah Schmidt, ${ }^{1}$ Oihane Horno, ${ }^{2,3}$ \\ Raoul-Martin Memmesheimer, ${ }^{2}$ and $\mathbb{C H}_{\text {Heinz Beck }}^{1,4}$ \\ ${ }^{1}$ Institute of Experimental Epileptology and Cognition Research, University of Bonn, 53105 Bonn, Germany, ${ }^{2}$ Neural Network Dynamics and Computation, \\ Institute of Genetics, University of Bonn, 53115 Bonn, Germany, ${ }^{3}$ Cortical Circuits Laboratory and Theoretical Neuroscience Laboratory, Champalimaud \\ Center for the Unknown, 1400-038 Lisbon, Portugal, and ${ }^{4}$ Deutsches Zentrum für Neurodegenerative Erkrankungen e.V., 53175 Bonn, Germany
}

Inhibitory interneurons, organized into canonical feedforward and feedback motifs, play a key role in controlling normal and pathological neuronal activity. We demonstrate prominent quantitative changes in the dynamics of feedback inhibition in a rat model of chronic epilepsy (male Wistar rats). Systematic interneuron recordings revealed a large decrease in intrinsic excitability of basket cells and oriens-lacunosum moleculare interneurons in epileptic animals. Additionally, the temporal dynamics of interneuron recruitment by recurrent feedback excitation were strongly altered, resulting in a profound loss of initial feedback inhibition during synchronous CA1 pyramidal activity. Biophysically constrained models of the complete feedback circuit motifs of normal and epileptic animals revealed that, as a consequence of altered feedback inhibition, burst activity arising in CA3 is more strongly converted to a CA1 output. This suggests that altered dynamics of feedback inhibition promote the transmission of epileptiform bursts to hippocampal projection areas.

Key words: circuit model; feedback inhibition; interneuron diversity; short-term plasticity; temporal lobe epilepsy

Significance Statement

We quantitatively characterized changes of the CA1 feedback inhibitory circuit in a model of chronic temporal lobe epilepsy. This study shows, for the first time, that dynamic recruitment of inhibition in feedback circuits is altered and establishes the cellular mechanisms for this change. Computational modeling revealed that the observed changes are likely to systematically alter CA1 input-output properties leading to (1) increased seizure propagation through CA1 and (2) altered computation of synchronous CA3 input.

\section{Introduction}

In the CNS, firing in neuronal ensembles is structured by the interaction of two fundamental categories of neurons: a majority of excitatory principal neurons and a minority $(\sim 10 \%-\% 15 \%)$ of inhibitory, mostly GABAergic interneurons. GABAergic interneurons mediate most of the shunting or hyperpolarizing inhibition in the adult brain, thereby powerfully controlling

\footnotetext{
Received Oct. 8, 2018; revised Aug. 28, 2019; accepted Sept. 4, 2019

Author contributions: L.P., R.-M.M., and H.B. designed research; L.P., C.K., 0.B., and 0.H. performed research; L.P., O.B., O.H., and R.-M.M. contributed unpublished reagents/analytic tools; L.P., C.K., O.B., and S.S. analyzed data; L.P. and C.K. wrote the first draft of the paper; O.B., R.-M.M., and H.B. edited the paper; O.B., R.-M.M., and H.B. wrote the paper.

This work was supported by Deutsche Forschungsgemeinschaft SFB 1089, German Federal Ministry of Education and Research via the Bernstein Network (Bernstein Award 2014,01GQ1501 and 01GQ1710), the BONFOR program of the University of Bonn Medical Center, the ERANET Neuron Grant EpiNet, the ERANET ebGLUNet, and the SPP 2041. We thank Margit Reitze, Olivia van Ray, and Marcus Bretz for excellent technical assistance.

The authors declare no competing financial interests.

*L.P., C.K., and 0.B., contributed equally to this work.

Correspondence should be addressed to Heinz Beck at heinz.beck@ukb.uni-bonn.de.

https://doi.org/10.1523/JNEUROSCI.2594-18.2019

Copyright $(2019$ the authors
}

neuronal input-output behavior. Interneurons display a staggering diversity, with a large number of subtypes (Freund and Buzsáki, 1996). These different interneuron types are organized into two fundamental categories of inhibition. Feedforward inhibition of pyramidal neurons results from activation of interneurons by the same synaptic pathway that excites the pyramidal neuron. Feedback inhibition, on the other hand, is recruited by pyramidal cell firing and activation of recurrent inhibitory microcircuits.

In the cortex and hippocampus, feedback inhibition exerts powerful control on output generation of pyramidal neurons (Miles, 1990; Ang et al., 2005). Different types of interneurons contribute to feedback inhibition, including interneuron classes mediating distal dendritic inhibition targeting the fine apical and basal branches of pyramidal neurons or proximal perisomatic inhibition. Intriguingly, somatically and dendritically targeting interneurons can be differentially recruited by different input frequencies, giving rise to timed and domain-specific inhibition of CA1 pyramidal neurons (Pouille and Scanziani, 2004). Although feedback inhibition in the CA1 region is known to pow- 
erfully influence CA1 excitability, its alteration in chronic epilepsy and the resulting consequences for CA1 input-output transformations are unknown. Indeed, effects on CA1 inputoutput transformation may be particularly relevant under conditions of epileptiform activity of the upstream CA3 region.

We have therefore examined the function of feedback inhibitory circuits in the normal and epileptic hippocampus. We find a pronounced change in the dynamics of inhibition of CA1 neurons that is rooted in both synaptic and intrinsic changes within the feedback circuit: Normally, synchronous activity of CA1 pyramidal cells recruits initially strong feedback inhibition that shows use-dependent depression. In contrast, initial inhibition is strongly reduced in chronic epilepsy. A biophysically constrained computational model suggests that these changed properties of feedback circuits promote the transmission of synchronous activity from CA3 via CA1 to other brain regions.

\section{Materials and Methods}

Pilocarpine model of epilepsy. Briefly, male Wistar rats (150-180 g, Charles River) were injected intraperitoneally with pilocarpine hydrochloride $(340 \mathrm{mg} / \mathrm{kg}$ body weight, Sigma-Aldrich), $30 \mathrm{~min}$ following a subcutaneous injection of $1 \mathrm{mg} / \mathrm{kg}$ scopolamine methyl nitrate (SigmaAldrich) to reduce peripheral cholinergic side effects. Within $60 \mathrm{~min}$ of injection, $30 \%-50 \%$ of the animals developed a limbic status epilepticus (SE) that was terminated by injection of $\sim 1 \mathrm{ml}$ diazepam $40 \mathrm{~min}$ after $\mathrm{SE}$ onset ( $4 \mathrm{mg} / \mathrm{kg}$ s.c., $0.5 \%$, Ratiopharm). In animals that did not develop SE after the first injection of pilocarpine, a second, identical injection of pilocarpine was administered. Following SE, rats received a single subcutaneous injection of $1 \mathrm{ml}$ Ringer's solution and $1 \mathrm{ml}$ glucose-solution and were kept in separate cages. Animals were video-monitored for $7 \mathrm{~d}$ starting $17 \mathrm{~d}$ after injection. Only rats displaying at least one spontaneous seizure were included in this study. Experiments were conducted 4-8 weeks following experimentally induced SE. Sham-control animals were treated in an identical manner but were injected with saline instead of pilocarpine. Data from a subset of cells in the sham-control condition have already been used in Pothmann et al. (2014). Specifically, of the morphologically identified interneuron subtypes (see below), data from 7 of 13 basket cells (BCs), 6 of 15 proximal dendritic cells (PDs), and 8 of 15 oriens-lacunosum moleculares (OLMs) were previously used.

Slice preparation and patch-clamp recording. Rats were deeply anesthetized with $1.5 \mathrm{ml}$ xylacinhydrochloride (2\%, Bayer) and $0.5 \mathrm{ml}$ ketamine hydrochloride (10\%, Pfizer), transcardially perfused with ice-cold preparation solution containing the following (in $\mathrm{mm}$ ): $60 \mathrm{NaCl}, 100$ sucrose, $2.5 \mathrm{KCl}, 1.25 \mathrm{NaH}_{2} \mathrm{PO}_{4}, 26 \mathrm{NaHCO}_{3}, 1 \mathrm{CaCl}_{2}, 5 \mathrm{MgCl}_{2}$, and 20 D-glucose (equilibrated with $95 \% \mathrm{O}_{2} / 5 \% \mathrm{CO}_{2}$ ). Transverse 300 - $\mu$ m-thick hippocampal slices were prepared on a vibratome (Microm HM $650 \mathrm{~V}$, Thermo Fisher Scientific). After equilibration for $30 \mathrm{~min}$ at $35^{\circ} \mathrm{C}$ in preparation solution, slices were transferred to ACSF containing the following (in mM): $125 \mathrm{NaCl}, 3 \mathrm{KCl}, 1.25 \mathrm{NaH}_{2} \mathrm{PO}_{4}, 26 \mathrm{NaHCO}_{3}, 2.6 \mathrm{CaCl}_{2}, 1.3$ $\mathrm{MgCl}_{2}$ and 15 D-glucose (equilibrated with $95 \% \mathrm{O}_{2}$ and $5 \% \mathrm{CO}_{2}$ ) and stored at room temperature.

For recordings, slices were transferred to a submerged chamber perfused with ACSF, mounted on the stage of an upright microscope (Axioscope 2 FS, Carl Zeiss), and cells were visualized with infrared oblique illumination optics and a water-immersion objective (60×, $0.9 \mathrm{NA})$. Somatic whole-cell recordings of interneurons or pyramidal neurons in the CA1 region were obtained with an Axopatch 200B amplifier (Molecular Devices), a Multiclamp 700 B amplifier (Molecular Devices), or a BVC-700 amplifier (Dagan). Data were filtered at $10 \mathrm{kHz}$ and sampled at $100 \mathrm{kHz}$ with a Digidata 1440 interface controlled by pClamp Software (Molecular Devices). Recording electrodes were made from thick-walled borosilicate glass capillaries (GB 150F 8P, Science Products) on a vertical puller (PP-830, Narishige). Recording pipettes for whole-cell recordings had a resistance of 3-6 $\mathrm{M} \Omega$ and were filled with the following (in $\mathrm{mm}$ ): $140 \mathrm{~K}$-gluconate, 5 HEPES, 0.16 EGTA, $0.5 \mathrm{MgCl}_{2}$, 5 phosphocreatine, and $0.3 \%$ biocytin, $\mathrm{pH} 7.3$ (280-290 mOsm). Series resistance ranged from 10 to $25 \mathrm{M} \Omega$ and was compensated with bridge balance circuitry in current clamp. The calculated liquid junction potential was $-15 \mathrm{mV}$, and membrane potential values were corrected accordingly. Interneurons or pyramidal cells were visually identified under infrared difference interference contrast optics, and further characterized functionally as well as morphologically by biocytin labeling and reconstruction (see Morphological reconstruction). All experiments were performed at $31^{\circ} \mathrm{C}-32^{\circ} \mathrm{C}$ either via an inline solution heater (TC324B, Warner Instruments) or a temperature controllable bath chamber (Temperature controller III, Luigs and Neumann). All animal experiments were conducted in accordance with the guidelines of the Animal Care and Use Committee of the University of Bonn.

Analysis of intrinsic properties. The input resistance $\mathrm{R}_{\mathrm{in}}$ was calculated from voltage deflections over current injections ranging from -50 to 50 pA with a linear fit (IGOR PRO, Wavemetrics). The membrane time constant was estimated using negative current injections and a standard exponential fit (Clampfit 10.2, Molecular Devices). The properties of postsynaptic currents (PSCs)/postsynaptic potentials (PSPs) were analyzed from an average of $\sim 10$ sweeps. Input-output properties were assessed by applying successively increasing $1 \mathrm{~s}$ current pulses up to $800 \mathrm{pA}$ $(0,10,20,30,40,60,80,100,125,150, \ldots, 775,800 \mathrm{pA})$. For analysis of single action potential (AP) properties, traces with two APs in the first 50 ms of current injection were selected and the first AP analyzed. The AP threshold was determined as the voltage at which the second derivative of the voltage trace peaked. The AP amplitude was determined as the difference between threshold and peak voltage, and the halfwidth was the width at half this amplitude. The sag value was calculated as the difference between peak and steady-state voltage deflection at the current injection at which steady-state voltage was $-75 \mathrm{mV}$ (i.e., $10-15 \mathrm{mV}$ below baseline). Neuronal input-output properties were analyzed only in the subset of cells where all current steps were recorded. The maximal firing rate was determined as the maximally obtained rate with injections up to $800 \mathrm{pA}$. The current for half-maximal firing was determined through sigmoidal fits. The rheobase current was determined as the first current injection eliciting an AP.

Analysis of feedback inhibitory circuit. For activation of CAl feedback microcircuits, we stimulated CA1 axons by placing a bipolar steel electrode (FHC) into the alveus adjacent to the subiculum and applying biphasic charge neutral pulses with a duration of $0.1 \mathrm{~ms}$ (Stimulus isolator: A-M Systems, model 2100). This stimulation leads to antidromic activation of CA1 axons and recruitment of feedback inhibitory circuits. To prevent a direct monosynaptic excitation or inhibition, a cut was made at the CA1-subiculum border through strata lacunosummoleculare, radiatum, pyramidale, and oriens with only the alveus left intact (Pouille and Scanziani, 2004). A second cut was made at the CA1/ CA3 border to limit spontaneous activity in CA1 neurons.

Feedback EPSPs were recorded in inhibitory interneurons to monitor temporal summation. Stimulation strength was set according to the following two criteria (identically in sham-control and epileptic animals): (1) the stimulation should not elicit APs, to allow proper estimation of the EPSP amplitudes; and (2) stimulation strength should be sufficiently large to elicit reliable EPSPs. The stimulation amplitudes were systematically varied at the beginning of each experiment until a stimulation strength that matched both criteria was found. The resultant stimulation strengths did not differ between sham-control and epileptic animals (mean \pm SD: $156 \pm 78$ and $186 \pm 96 \mu$ A for cells from sham and epileptic slices respectively; $n=34$ and $n=37$, respectively; $p_{(t=1.435, \mathrm{df}=69)}=$ 0.156 , unpaired Student's $t$ test). Only interneurons that could be unambiguously identified based on morphology were included ( 81 of 171 , see below). IPSCs were recorded from pyramidal neurons that result from activation of feedback interneurons. In these experiments, PSCs (rather than PSPs) were measured because they allowed to better distinguish individual IPSCs in train stimulations. IPSC amplitudes were measured from pre-stimulus-train baseline, except for in paired pulse analysis, where it was measured from the local pre-IPSC minimum. To isolate the GABAergic IPSC unequivocally, the $\mathrm{GABA}_{\mathrm{A}}$ receptor antagonist gabazine (SR 95531 hydrobromide, $10 \mu \mathrm{M}$, Tocris Bioscience) was applied at the end of all stimulation experiments, and the IPSC component was isolated by subtraction. For these recordings, pyramidal neurons were clamped at $-65 \mathrm{mV}$. This procedure usually revealed no or only a minor 
excitatory component, indicating that contamination by Schaffer collateral or recurrent excitatory CA1 input is negligible in this paradigm (Pothmann et al., 2014).

During all stimulation experiments, the $\mathrm{GABA}_{\mathrm{B}}$ blocker CGP 52432 (500 nM, Tocris Bioscience) was present in the bath solution.

Morphological reconstruction. Slices containing biocytin filled cells $(0.3 \%)$ were incubated at $4^{\circ} \mathrm{C}$ in PFA (4\%) dissolved in 0.1 PB, pH 7.4. For biocytin staining, slices were washed in $0.15 \mathrm{M}$ PBS and permeabilized for 30 min with Triton X-100 ( $0.4 \%$ in PBS). Slices were then incubated for $2 \mathrm{~h}$ in Streptavidin AlexaFluor-488 (1:500 in PBS and Triton X-100 $0.1 \%$ ). Subsequently, sections were washed in $0.1 \mathrm{M} \mathrm{PB} 3$ times for $5 \mathrm{~min}$, and mounted with Aqua-Poly/Mount. Filled neurons were scanned on a confocal microscope and reconstructed from $z$ stacks ( $40 \times$ magnification, step size: $1-2.5 \mu \mathrm{m}$ ).

Cell classification and morphological analysis. BCs were identified via their distinctive axon distribution in the pyramidal cell layer. PD cells comprise cells whose axon ramified within stratum oriens and stratum radiatum (and potentially also stratum pyramidale). OLM cells were identified based on soma location in stratum oriens and visible axon in stratum lacunosum moleculare. Of 171 recorded cells identified as interneurons based on electrophysiological properties and soma location, 81 could be thus visually identified ( $n=43$ and $n=37$ cells in sham and epileptic animals, respectively). Only these 81 cells were included in the analyses in the manuscript. In 30 of these, biocytin filling was sufficient to allow morphological analysis $(n=12$ and $n=18$ cells in sham and epileptic animals, respectively). Image processing and morphological analysis were performed using ImageJ software. Cells were maxprojected into a single image, and the area covered by soma, dendrites, and axon in each hippocampal sublayer was quantified as the smallest surrounding polygon. Additionally, dendritic trees were traced and analyzed using simple neurite tracer (Longair et al., 2011). Mean bouton density was quantified on five random terminal axon branches per cell as the number of putative boutons divided by the branch length. In a subset of cells with very good biocytin filling, we were able to trace the dendritic and axonal arbors in 3D ( $n=17$ cells). These will be submitted to www. neuromorpho.org.

Statistical analysis. Average values in the text and figures are expressed as mean \pm SEM unless stated otherwise. Box plots represent values for median, 25th and 75th percentile, as well as minimum and maximum. For statistical analysis, Mann-Whitney $U$ test, Wilcoxon signed-rank test, Student's $t$ test, or ANOVA was used as indicated in the text. Test statistic and degrees of freedom are given as subscript for each $p$ value (for ANOVA as $\mathrm{df}=\mathrm{DFn}, \mathrm{DFd}$ ).

Computational model. In the following, we give a detailed description of the models we used for the basket and pyramidal cells and their synapses and of the model of the complete feedback motif. Furthermore, we describe the fitting procedure we used. Model and fitting were implemented using MATLAB R2018a (The MathWorks).

Model of BCs. To model the excitation of the BC population, we used a nonlinear leaky integrator neuron model with a current-based synapse that exhibits short-term plasticity (STP). To model the STP, we used a modified version of the model introduced by Markram et al. (1998).

Specifically, the state of the population is represented by an effective average membrane voltage (or by the membrane potential of one representative neuron) $V_{\mathrm{BC}}(t)$, whose dynamics are governed by the following:

$$
\tau_{\mathrm{d}, \mathrm{BC}} \frac{d V_{\mathrm{BC}}}{\mathrm{d} t}(t)=-V_{B C}(t)+\lambda V_{B C}^{2}(t)+I_{\mathrm{e}, \mathrm{BC}}(t)
$$

where $\tau_{\mathrm{d}, \mathrm{BC}}$ is the membrane time constant, $\lambda$ specifies the strength of the nonlinearity, and $I_{\mathrm{e}, \mathrm{BC}}(t)$ is the input current. Since the BCs do not spike in the experimental setting, we did not include a term covering spikes and resets in the model. The input current describes the effect of the synaptic input on the BC population. For the fit to the experimental data, we assume that the population of neurons receives similar inputs from a number of synapses that are excited in the same way by the stimulation. The total input is the linear superposition of the individual synaptic currents. Like a single synaptic current, it obeys the following:

$$
\tau_{\mathrm{e}, \mathrm{BC}} \frac{\mathrm{d} I_{\mathrm{e}, \mathrm{BC}}}{\mathrm{d} t}(t)=-I_{\mathrm{e}, \mathrm{BC}}(t)+\tau_{\mathrm{e}, \mathrm{BC}} \hat{I}_{\mathrm{e}, \mathrm{BC}} \tilde{I}_{\mathrm{e}, \mathrm{BC}} \mathcal{u}_{\mathrm{e}, \mathrm{BC}}\left(t^{-}\right) x_{\mathrm{e}, \mathrm{BC}}\left(t^{-}\right) \sum_{t_{\mathrm{sp}}} \delta\left(t-t_{\mathrm{sp}}\right),
$$

where $\tau_{\mathrm{e}, \mathrm{BC}}$ is the synaptic time constant, $u_{\mathrm{e}, \mathrm{BC}}\left(t^{-}\right)$is the fraction of available neurotransmitters released from the readily releasable pool when a spike arrives at the synapse, $x_{\mathrm{e}, \mathrm{BC}}\left(t^{-}\right)$is the current fraction of available neurotransmitters in the readily releasable pool when a spike arrives at the synapse, $\hat{I}_{\mathrm{e}, \mathrm{BC}}$ specifies the impact strength of the released fraction of neurotransmitters, and $\tilde{I}_{\mathrm{e}, \mathrm{BC}}$ is a normalization constant, which ensures that the EPSP amplitude is $\hat{I}_{\mathrm{e}, \mathrm{BC}}$ if $\lambda=0$ and if $u_{\mathrm{e}, \mathrm{BC}}\left(t^{-}\right)$ and $x_{\mathrm{e}, \mathrm{BC}}\left(t^{-}\right)$assume their asymptotic values (as after an infinitely long preceding interspike interval). The $t_{\text {sp's }}$ are the times of spike arrival at the synapse, set according to the considered stimulation protocol. $x_{\mathrm{e}, \mathrm{BC}}(t)$ introduces short-term synaptic depression and is determined by the following:

$\tau_{\mathrm{RRP}, \mathrm{e}, \mathrm{BC}} \frac{d x_{\mathrm{e}, \mathrm{BC}}}{\mathrm{d} t}(t)=1-x_{\mathrm{e}, \mathrm{BC}}(t)-\tau_{\mathrm{RRP}, \mathrm{e}, \mathrm{BC}} u_{\mathrm{e}, \mathrm{BC}}\left(t^{-}\right) x_{\mathrm{e}, \mathrm{BC}}\left(t^{-}\right) \sum_{t_{\mathrm{sp}}} \delta\left(t-t_{\mathrm{sp}}\right)$,

where $\tau_{\mathrm{RRP}, \mathrm{e}, \mathrm{BC}}$ is the time constant of the depression. $u_{\mathrm{e}, \mathrm{BC}}(t)$ introduces short-term synaptic facilitation and is determined by the following:

$$
\begin{aligned}
\tau_{\mathrm{fa}, e, \mathrm{BC}} \frac{\mathrm{d} u_{\mathrm{e}, \mathrm{BC}}}{\mathrm{d} t}(t)=u_{0, e, \mathrm{BC}} & -u_{\mathrm{e}, \mathrm{BC}}(t) \\
& +\tau_{\mathrm{fac}, \mathrm{e}, \mathrm{BC}} u_{\mathrm{f}, \mathrm{e}, \mathrm{BC}}\left(1-u_{\mathrm{e}, \mathrm{BC}}\left(t^{-}\right)\right) \sum_{t_{\mathrm{sp}}} \delta\left(t-t_{\mathrm{sp}}\right)
\end{aligned}
$$

Here, $\tau_{\text {fac,e,BC }}$ is the time constant of the facilitation, $u_{0, e, \mathrm{BC}}$ is the asymptotic release fraction corresponding to the release fraction of vesicles after an infinitely long preceding interspike interval, and $u_{\mathrm{f}, \mathrm{e}, \mathrm{BC}}$ characterizes the size of the jump toward 1 upon spike arrival. In the original version of this model (Markram et al., 1998), the jump size was also set to $u_{0, \mathrm{e}, \mathrm{BC}}$, such that it served a double role (jump size and equilibrium fraction of released neurotransmitters), which does not seem justified for our data. All parameter values were determined through the fitting procedure described below for both the control and epileptic case.

Model of pyramidal cells. To model the pyramidal cell feedback inhibition, we first computed the effective membrane potential of the BC population in response to the stimuli with a model extending the model we used to reproduce the $\mathrm{BC}$ data. The extended model incorporates a term that covers resets after spiking, depending on an estimate of the population-averaged firing rate of a single BC. We then used a synapse model, which again included STP, to determine the inhibitory current in the pyramidal cells as a function of the rate of the $\mathrm{BC}$ population.

We computed the input current of the mean BC with Equations 2-4. To account for the reset of the membrane potential after spikes, we extended Equation 1 to the following:

$$
\tau_{\mathrm{d}, \mathrm{BC}} \frac{d V_{\mathrm{BC}}}{\mathrm{d} t}(t)=-V_{\mathrm{BC}}(t)+\lambda V_{B C}^{2}(t)+I_{\mathrm{e}, \mathrm{BC}}(t)-\tau_{\mathrm{d}, \mathrm{BC}} V_{\mathrm{BC}}(t) r_{\mathrm{BC}}(t) .
$$

Here, $r_{\mathrm{BC}}(t)$ is the estimate of the population-averaged instantaneous firing rate of the $\mathrm{BCs}$. It is given by the following:

$$
r_{\mathrm{BC}}(t)=\hat{r}_{\mathrm{BC}} \log \left(1+\exp \left(\frac{V_{\mathrm{BC}}(t)-V_{\mathrm{th}, \mathrm{BC}}}{V_{\mathrm{w}, \mathrm{BC}}}\right)\right),
$$

where $V_{\mathrm{th}, \mathrm{BC}}$ is the firing threshold, $V_{\mathrm{w}, \mathrm{BC}}$ specifies the softness of the firing threshold, and $\hat{r}_{\mathrm{BC}}$ determines how fast the firing rate increases with increasing $V_{\mathrm{BC}}(t)$. With the exception of $\hat{I}_{\mathrm{BC}}$, all parameters that occur already in Equations 1-4 were set to the values found by the fit of the $\mathrm{BC}$ model to the cell-averaged $\mathrm{BC}$ data. We had to newly fit $\hat{I}_{\mathrm{BC}}$, since the level of stimulation of BCs was different (higher) in the current paradigm, such that they received a suprathreshold input. Furthermore, instead of fitting the parameters occurring in Equation 6 to the data, we 
preset $V_{\text {th }, \mathrm{BC}}=6 \mathrm{mV}, V_{\mathrm{w}, \mathrm{BC}}=0.2 \mathrm{mV}$, and $\hat{r}_{\mathrm{BC}}=20 \mathrm{~Hz}$ as the quality of the fit to the pyramidal cell data is highly insensitive to the exact values of these parameters. This is because some of the parameters in the model have a similar effect on the output firing rate. For example, an increase of $\hat{I}_{\mathrm{BC}}$ and a decrease of $V_{\mathrm{th}, \mathrm{BC}}$ both primarily led to a higher firing rate of the BCs. The fixed parameters could thus be chosen within a broad range where the overall activity of the BCs during the stimulation protocols is biologically plausible. In particular, while firing rates can be high during excursions of the voltage above threshold, such excursions are brief in our data, indicating the generation of only a few spikes.

Using the firing rate of the $\mathrm{BCs}$, we computed the inhibitory current in the pyramidal cells. The inhibitory current in the pyramidal cells $I_{\mathrm{i}, \mathrm{PY}}(t)$ is determined by the following:

$$
\tau_{\mathrm{i}, \mathrm{PY}} \frac{\mathrm{d} I_{\mathrm{i}, \mathrm{PY}}}{\mathrm{d} t}(t)=-I_{\mathrm{i}, \mathrm{PY}}(t)+\tau_{\mathrm{i}, \mathrm{PY}} \hat{\mathrm{I}}_{\mathrm{i}, \mathrm{PY}} \tilde{I}_{\mathrm{i}, \mathrm{PY}} u_{\mathrm{i}, \mathrm{PY}}(t) x_{\mathrm{i}, \mathrm{PY}}(t) r_{\mathrm{BC}}(t),
$$

where $\tau_{\mathrm{i}, \mathrm{PY}}$ is the synaptic time constant, $u_{\mathrm{i}, \mathrm{PY}}(t)$ is the release fraction of the neurotransmitters, $x_{\mathrm{i}, \mathrm{PY}}(t)$ is the fraction of available neurotransmitters in the readily releasable pool, $\hat{I}_{\mathrm{i}, \mathrm{PY}}$ specifies the impact strength of the released fraction of neurotransmitters, and $\tilde{I}_{\mathrm{i}, \mathrm{PY}}$ is a normalization constant, which ensures that the IPSC amplitude is $\hat{I}_{\mathrm{i}, \mathrm{PY}}$ if $u_{\mathrm{i}, \mathrm{PY}}(t)$ and $x_{\mathrm{i}, \mathrm{PY}}(t)$ assume their asymptotic values. $x_{\mathrm{i}, \mathrm{PY}}(t)$ introduces short-term depression and is determined by the following:

$$
\tau_{\mathrm{RRP}, \mathrm{i}, \mathrm{PY}} \frac{\mathrm{d} x_{\mathrm{i}, \mathrm{PY}}}{\mathrm{d} t}(t)=1-x_{\mathrm{i}, \mathrm{PY}}(t)-\tau_{\mathrm{RRP}, \mathrm{i}, \mathrm{PY}} u_{\mathrm{i}, \mathrm{PY}}(t) x_{\mathrm{i}, \mathrm{PY}}(t) r_{\mathrm{BC}}(t),
$$

where $\tau_{\mathrm{RRP}, \mathrm{i}, \mathrm{PY}}$ is the time constant of the depression. $u_{\mathrm{i}, \mathrm{PY}}(t)$ introduces short-term facilitation and is determined by the following:

$$
\tau_{\mathrm{fac}, \mathrm{i}, \mathrm{PY}} \frac{\mathrm{d} u_{\mathrm{i}, \mathrm{PY}}}{\mathrm{d} t}(t)=u_{0, \mathrm{i}, \mathrm{PY}}-u_{\mathrm{i}, \mathrm{PY}}(t)+\tau_{\mathrm{fac}, \mathrm{i}, \mathrm{PY}} u_{\mathrm{f}, \mathrm{i}, \mathrm{PY}}\left(1-u_{\mathrm{i}, \mathrm{PY}}(t)\right) r_{\mathrm{BC}}(t) .
$$

Here, $\tau_{\text {fac,i,PY }}$ is the time constant of the facilitation, $u_{0, \mathrm{i}, \mathrm{PY}}$ is the asymptotic release fraction corresponding to the release fraction of vesicles after an infinitely long preceding interval with no presynaptic activity, and $u_{\mathrm{f}, \mathrm{i}, \mathrm{PY}}$ characterizes the increase of the release fraction of the neurotransmitters. All so far unspecified parameter values were determined through the fitting procedure described below for both the control and epileptic case.

Model of the complete feedback motif. To determine how the complete inhibitory feedback loop reacts to input, we combined the basket and pyramidal cell models. The two population models are recurrently connected and have firing rates $r_{\mathrm{BC}}(t)$ and $r_{\mathrm{PY}}(t)$. The pyramidal cell population additionally receives as excitatory input a firing rate $r_{\mathrm{IN}}(t)$ from CA3. We computed the firing rates of the basket and pyramidal cells using the experimentally measured relations between input current and firing rate (see Fig. $4 B$ ).

Pyramidal cells. The firing rate of an average pyramidal neuron as a function of the input current $I_{\mathrm{PY}}(t)$ is given by the following:

$$
r_{\mathrm{PY}}(t)=r_{0, \mathrm{PY}}+\frac{r_{1, \mathrm{PY}}}{1+\exp \left(-\frac{I_{\mathrm{PY}}(t)-I_{\frac{2}{2} \mathrm{PY}}^{1}}{I_{\mathrm{w}, \mathrm{PY}}}\right)}
$$

For the control case, fitting the experimental data yielded the parameters $r_{0, \mathrm{PY}}=-1.60 \mathrm{~Hz}, r_{1, \mathrm{PY}}=23.03 \mathrm{~Hz}, I_{2}^{1} \mathrm{PY}=249.91 \mathrm{pA}$ and $I_{\mathrm{w}, \mathrm{PY}}=96.38$ $\mathrm{pA}$. For the epileptic case, we obtained $r_{0, \mathrm{PY}}=-1.74 \mathrm{~Hz}, r_{1, \mathrm{PY}}=31.63$ $\mathrm{Hz}, I_{\overline{2}}^{1} \mathrm{PY}=377.32 \mathrm{pA}$ and $I_{\mathrm{w}, \mathrm{PY}}=132.75 \mathrm{pA}$. The input current to the pyramidal cells is the sum of an excitatory current $I_{\mathrm{e}, \mathrm{PY}}(t)$ and an inhibitory current $-I_{\mathrm{i}, \mathrm{PY}}(t)$. As there are no experimental data available about the STP of the excitatory synapses between CA3 neurons and CA1 in epilepsy, we assumed for simplicity that this synapse generally does not exhibit STP. Thus, the excitatory current is governed by the following:

$$
\tau_{\mathrm{e}, \mathrm{PY}} \frac{\mathrm{d} I_{\mathrm{e}, \mathrm{PY}}}{\mathrm{d} t}(t)=-I_{\mathrm{e}, \mathrm{PY}}(t)+\tau_{\mathrm{e}, \mathrm{PY}} \hat{I}_{\mathrm{e}, \mathrm{PY}} r_{\mathrm{IN}}(t),
$$

where $\tau_{\mathrm{e}, \mathrm{PY}}$ is the synaptic time constant and $\hat{I}_{\mathrm{e}, \mathrm{PY}}$ is the coupling strength between the external input and the pyramidal cells. For both the control and the epileptic case, we set $\tau_{\mathrm{e}, \mathrm{PY}}=5 \mathrm{~ms}$ in rough accordance to experimental data (Spruston et al., 1995; Smith et al., 2003) and $\hat{I}_{\mathrm{e}, \mathrm{PY}}=2000$ $\mathrm{pA}$. We selected the values of this and all further coupling strengths to achieve biologically plausible activity levels, with the same coupling strength in the control and epileptic case. To determine the inhibitory current $I_{\mathrm{i}, \mathrm{PY}}(t)$, we used Equations 7-9 with the parameters set to the values obtained from the fit to the cell-averaged pyramidal cell data, except for $\hat{I}_{\mathrm{i}, \mathrm{PY}}$, as the inhibition is not a result of external stimulation anymore but models biological neuronal activity. Specifically, we set $\hat{I}_{\mathrm{i}, \mathrm{PY}}$ $=1000 \mathrm{pA}$ in both the control and the epileptic case. We used the same value for both cases as the fit to the cell-averaged pyramidal cell data also yielded similar values for $\hat{I}_{\mathrm{i}, \mathrm{PY}}$ in the control and epileptic case.

$B C$ s. The firing rate of an average $\mathrm{BC}$ as a function of the input current $I_{\mathrm{e}, \mathrm{BC}}(t)$ is given by the following:

$$
r_{\mathrm{BC}}(t)=r_{0, \mathrm{BC}}+\frac{r_{1, \mathrm{BC}}}{1+\exp \left(\frac{I_{\mathrm{e}, \mathrm{BC}}(t)-I_{2}^{1}, \mathrm{BC}}{I_{\mathrm{w}, \mathrm{BC}}}\right)}
$$

For the control case, we obtained the parameters $r_{0, \mathrm{BC}}=-12.15 \mathrm{~Hz}$, $r_{1, \mathrm{BC}}=141.02 \mathrm{~Hz}, I_{\overline{2}}^{1} \mathrm{BC}=383.44 \mathrm{pA}$ and $I_{\mathrm{w}, \mathrm{BC}}=162.37 \mathrm{pA}$, and for the epileptic case the parameters $r_{0, \mathrm{BC}}=-1.09 \mathrm{~Hz}, r_{1, \mathrm{BC}}=104.58 \mathrm{~Hz}, I_{\overline{2}}^{1} \mathrm{BC}=$ $487.64 \mathrm{pA}$ and $I_{\mathrm{w}, \mathrm{BC}}=107.16 \mathrm{pA}$. We computed the input current $I_{\mathrm{e}, \mathrm{BC}}(t)$ similarly to Equations 2-4. Specifically, we replaced $\Sigma_{t_{\mathrm{sp}}} \delta$ $\left(t-t_{\mathrm{sp}}\right)$ with $r_{\mathrm{PY}}(t)$ in Equations $2-4$ and additionally replaced $\hat{I}_{\mathrm{e}, \mathrm{BC}} \tilde{I}_{\mathrm{e}, \mathrm{BC}}$ with $\frac{\hat{I}_{e, \mathrm{BC}}}{u_{0, \mathrm{e}, \mathrm{BC}}}$ in Equation 2. The parameters are set to the values obtained from the fit to the cell-averaged $\mathrm{BC}$ data, except for the coupling strength $\hat{I}_{\mathrm{e}, \mathrm{BC}}$, for the same reason as before. We set $\hat{I}_{\mathrm{e}, \mathrm{BC}}=15000 \mathrm{pA}$ in the control case and $\hat{I}_{\mathrm{e}, \mathrm{BC}}=10000 \mathrm{pA}$ in the epileptic case. We chose these different values as the coupling strength was $\sim 1.5$ times higher in the control compared with the epileptic case for the fit to the cell-averaged $\mathrm{BC}$ data. We extracted the coupling strength from the fit to the cellaveraged $\mathrm{BC}$ data by dividing $\hat{\mathrm{I}}_{\mathrm{e}, \mathrm{BC}} \tilde{\mathrm{I}}_{\mathrm{e}, \mathrm{BC}}$ by the mean input resistance of the cells used for the fitting.

Fitting procedure. We fitted the BC model to the voltage traces measured in $\mathrm{BCs}$ and the pyramidal cell model to the current traces measured in pyramidal cells during both the $50 \mathrm{~Hz}$ and theta burst protocol. As described above, we used the fit to the cell-averaged data to describe the behavior of the cell populations as a whole, which is the characteristic relevant for modeling the feedback circuit. The goal of the fitting procedure was to minimize following model error:

$$
E=\sqrt{\frac{1}{N_{50}} \sum_{t_{50}}\left(Y_{50}\left(t_{50}\right)-X_{50}\left(t_{50}\right)\right)^{2}+\frac{1}{N_{T B}} \sum_{t_{\mathrm{TB}}}\left(Y_{\mathrm{TB}}\left(t_{\mathrm{TB}}\right)-X_{\mathrm{TB}}\left(t_{\mathrm{TB}}\right)\right)^{2}},
$$

where $t_{50}$ and $t_{\mathrm{TB}}$ are the time steps in the $50 \mathrm{~Hz}$ and theta burst protocol and $N_{50}$ and $N_{\text {TB }}$ are their numbers. $Y_{50}\left(t_{50}\right)$ is the experimentally observed potential of the BCs (current in the pyramidal cells) at time step $t_{50}$ in the $50 \mathrm{~Hz}$ protocol and $X_{50}\left(t_{50}\right)$ is the corresponding value in our model. Likewise, $Y_{\mathrm{TB}}\left(t_{\mathrm{TB}}\right)$ is the experimentally observed potential of the BCs (current in the pyramidal cells) at time step $t_{\mathrm{TB}}$ in the theta burst protocol and $X_{\mathrm{TB}}\left(t_{\mathrm{TB}}\right)$ is the corresponding value in our model. To validate the fitted parameter combinations in the biologically plausible range of parameters, we generated error surfaces. This approach safeguards against local minima and allows to display the parameter region that yields good fits and its localization (see Fig. 7C,D).

The first step was to compute the model error on a regular grid in the parameter space. This grid was located in a region of biologically plausible parameter values (see Tables 4, 5). In the case of the BC model, this grid consisted of $10^{8}$ points, with 10 regularly spaced values for each of the 8 parameters; and in the case of the pyramidal cell model, it consisted of $13^{7}$ points, with 13 regularly spaced values for each of the 7 parameters (see Fig. $7 C, D$ ). In the second step, we first determined the local minima 
of the model error on this grid. Starting from each of the found minima, we then performed a localized grid search to further minimize the model error. Typically, the grid search yielded two parameter sets with similarly small fitting error, whose only substantial differences were that one of the sets exhibited no short-term facilitation whereas the other point exhibited a small amount of facilitation (e.g., large $u_{\mathrm{f}, \mathrm{e}, \mathrm{BC}}$ but small $\left.\tau_{\text {fac,e, BC }}\right)$. This indicates that facilitation is negligible. We thus chose the point corresponding to the simpler model without short-term facilitation for the further analysis.

Code accessibility: Code is available upon request.

\section{Results}

All canonical feedback circuits consist of the same key elements: excitatory connections from a principal cell population that synaptically recruit inhibitory interneurons, which then inhibit the principal cell population. We have systematically examined the properties of the key elements of feedback circuits in the hippocampal CA1 region, and how they change in chronic epilepsy.

\section{Altered activation of CA1 interneurons within feedback microcircuits}

We first determined the properties of feedback activation of three categories of CA1 interneurons targeting different areas of the somatodendritic axis of pyramidal cells (Fig. 1A,B). Only interneurons that could be unambiguously classified into one of these three categories based on their axon morphology were included in this study (see Materials and Methods). The first category consisted of BCs that innervate pyramidal cell somata (Fig. $1 A, B$, left; sham-control and epileptic animals, respectively, $n=13$ and $n=14)$. A second group included cells that target the proximal dendrites of pyramidal cells in stratum radiatum and oriens (i.e., bistratified cells) (Somogyi and Klausberger, 2005), as well as cells that additionally innervate stratum pyramidale (i.e., trilaminar cells) and were collectively termed PDs (Fig. $1 A, B ; n=15$ and $n=10$ ). Third, we examined OLM interneurons with somata located in stratum oriens and axonal projections innervating the distal pyramidal cell dendrites in stratum lacunosum moleculare (Fig. $1 A, B ; n=15$ and $n=14$ ). A subset of morphologically identified cells were sufficiently filled to allow a more detailed morphological analysis regarding the laminar distribution of axons and dendrites, and the axonal bouton density ( $\mathrm{Ta}-$ bles 1, 2; see Materials and Methods, reconstructions available at www.neuromorpho.org). However, although some effects were suggestive, we found no significant morphological differences

E

$\mathbf{F}$
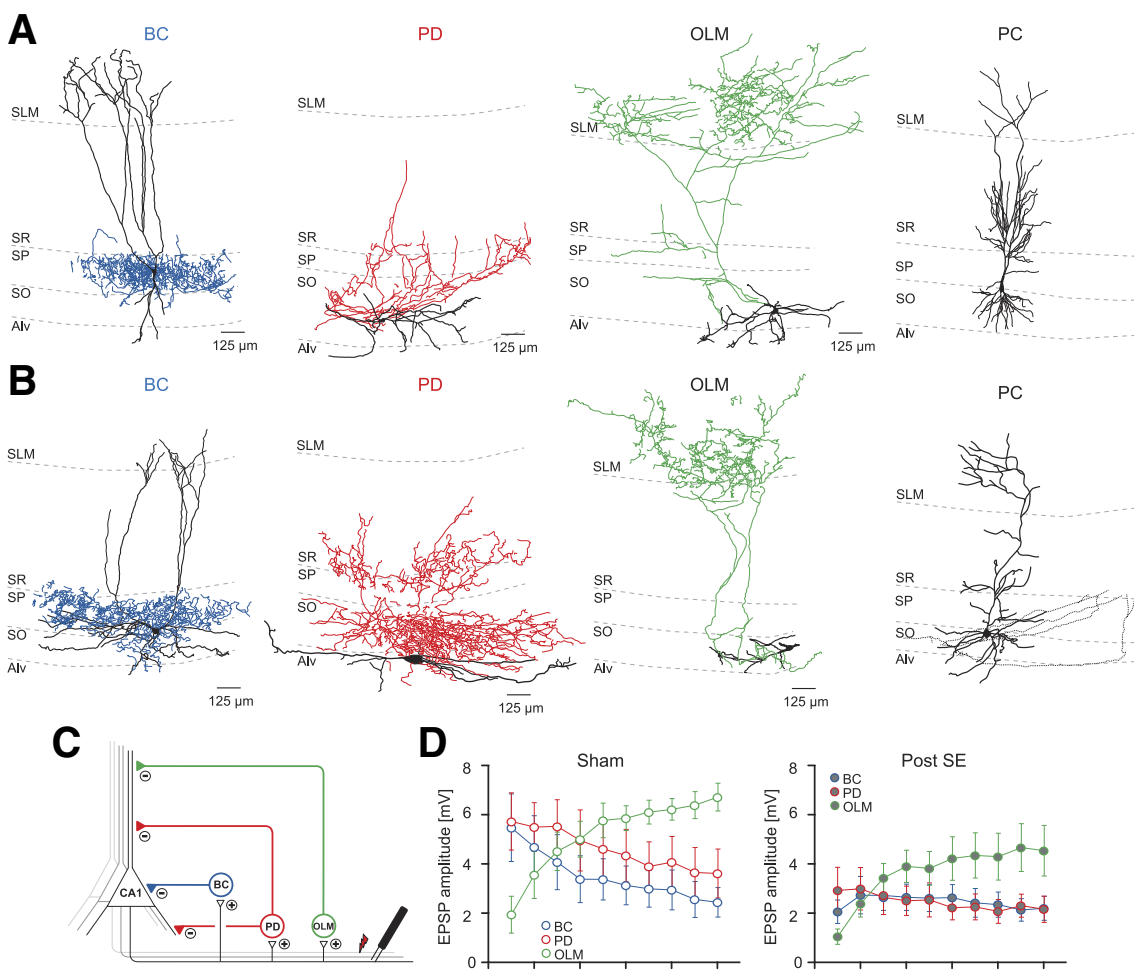

PD
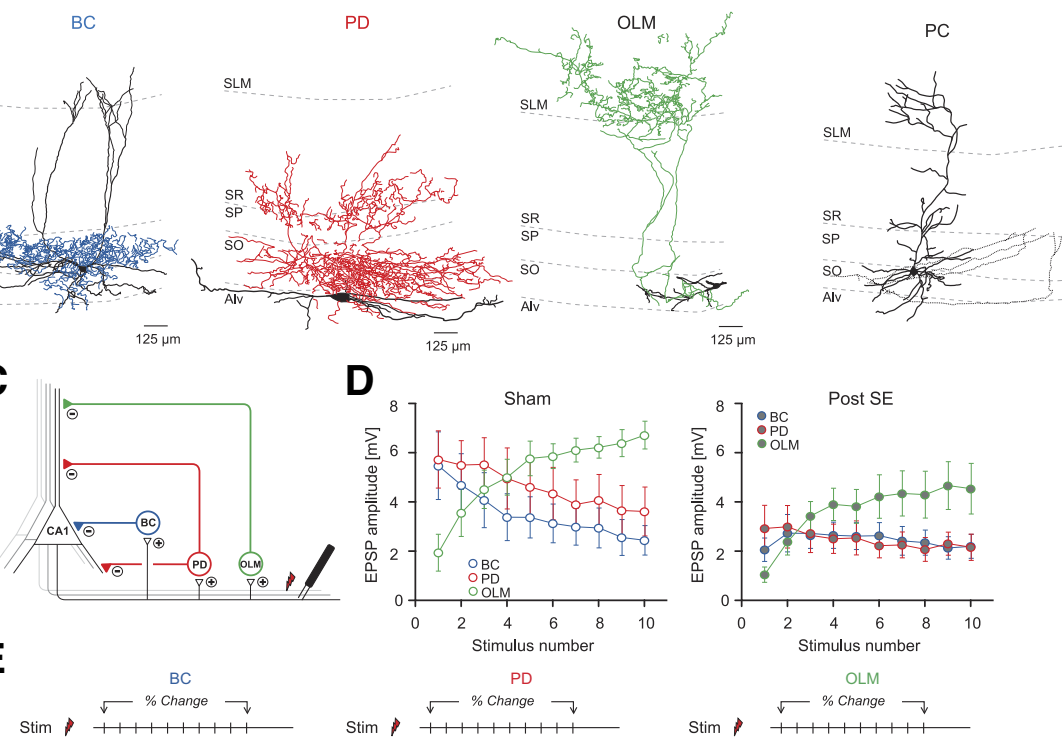

D
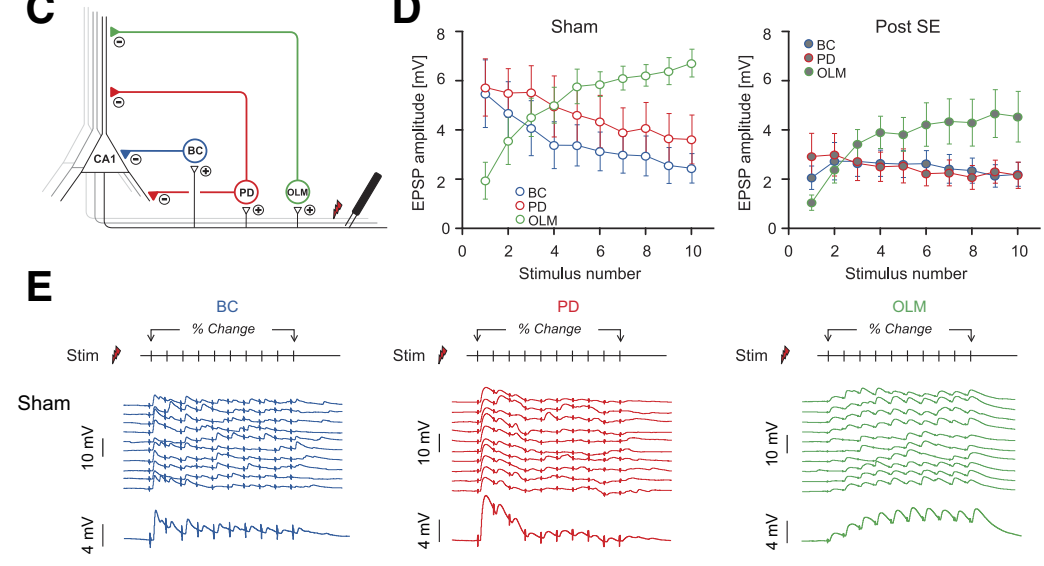

$\mathrm{PD}$
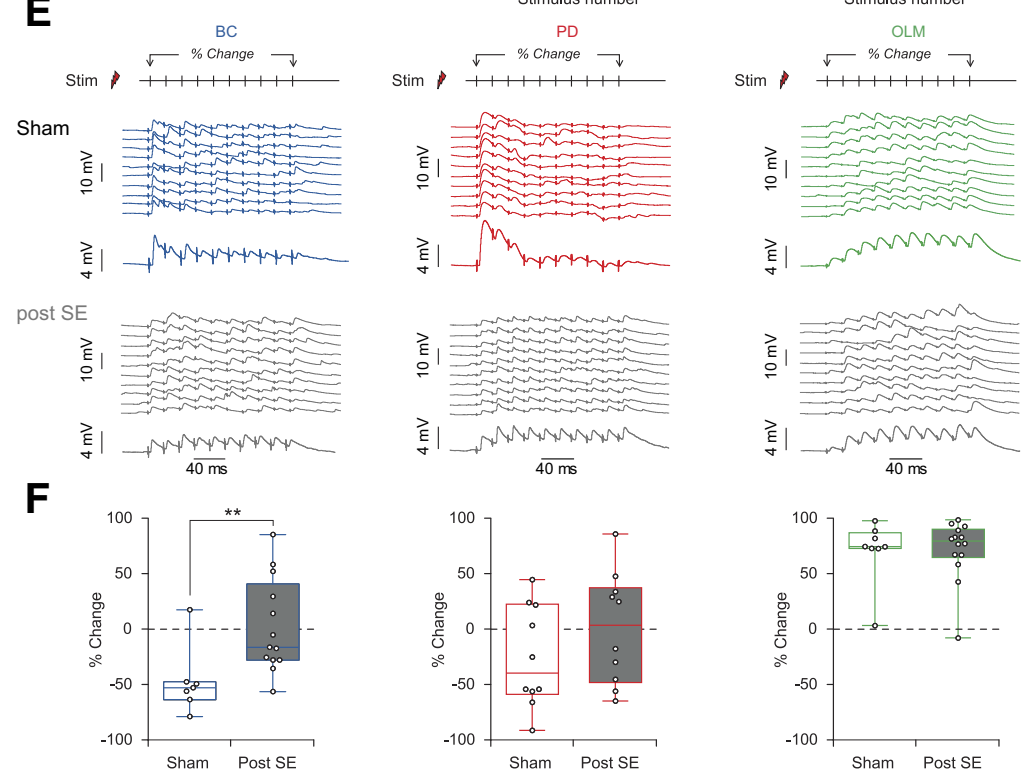

Figure 1. Epilepsy-associated changes in feedback recruitment of different interneuron types during a $50 \mathrm{~Hz}$ stimulus train. $\boldsymbol{A}$, $B$, Representative morphological reconstructions of $B C, P D$, and $0 L M$ interneurons, as well as pyramidal neurons following wholecell recording in sham-injected $(\boldsymbol{A})$ or epileptic animals (Post SE; $\boldsymbol{B}$ ). Axons are shown in blue, red, black, or green in the different types of neurons. Black represents the dendritic reconstruction. $\boldsymbol{C}$, Schematic diagram illustrating placement of the stimulus electrode into the alveus and recording of feedbackEPSPs in interneurons. D, Time course of feedback EPSPs elicited by a train of 10 stimuli at $50 \mathrm{~Hz}$ in BCs, PDs, and OLMs. Left, Sham-control animals. Right, Epileptic animals. E, Examples of feedback EPSPs elicited by $50 \mathrm{~Hz}$ train stimulation in BCS, PD cells, and OLM cells in sham-control (blue, red, and green, respectively) and epileptic animals (gray traces). For each condition and cell type, 10 single sweeps followed by their mean is shown. $\boldsymbol{F}$, Quantification of the dynamics of excitation onto the different interneuron types. The percent change in EPSP amplitude over the stimulus (first EPSP vs mean of the last three EPSPs) was calculated. Box plots represent median, 25th and 75th percentile, as well as minimum and maximum. Individual values are superimposed. $B(s ; p=0.008$ (Mann-Whitney $U$ test). between sham and epileptic animals within the available subsamples (Tables 1, 2). Finally, we also examined CA1 pyramidal neurons in sham-control and epileptic animals (Fig. $1 A, B$, rightmost panels; $n=13$ and $n=14$ ). 
Table 1. Morphological properties of interneurons in control and epileptic animals ${ }^{a}$

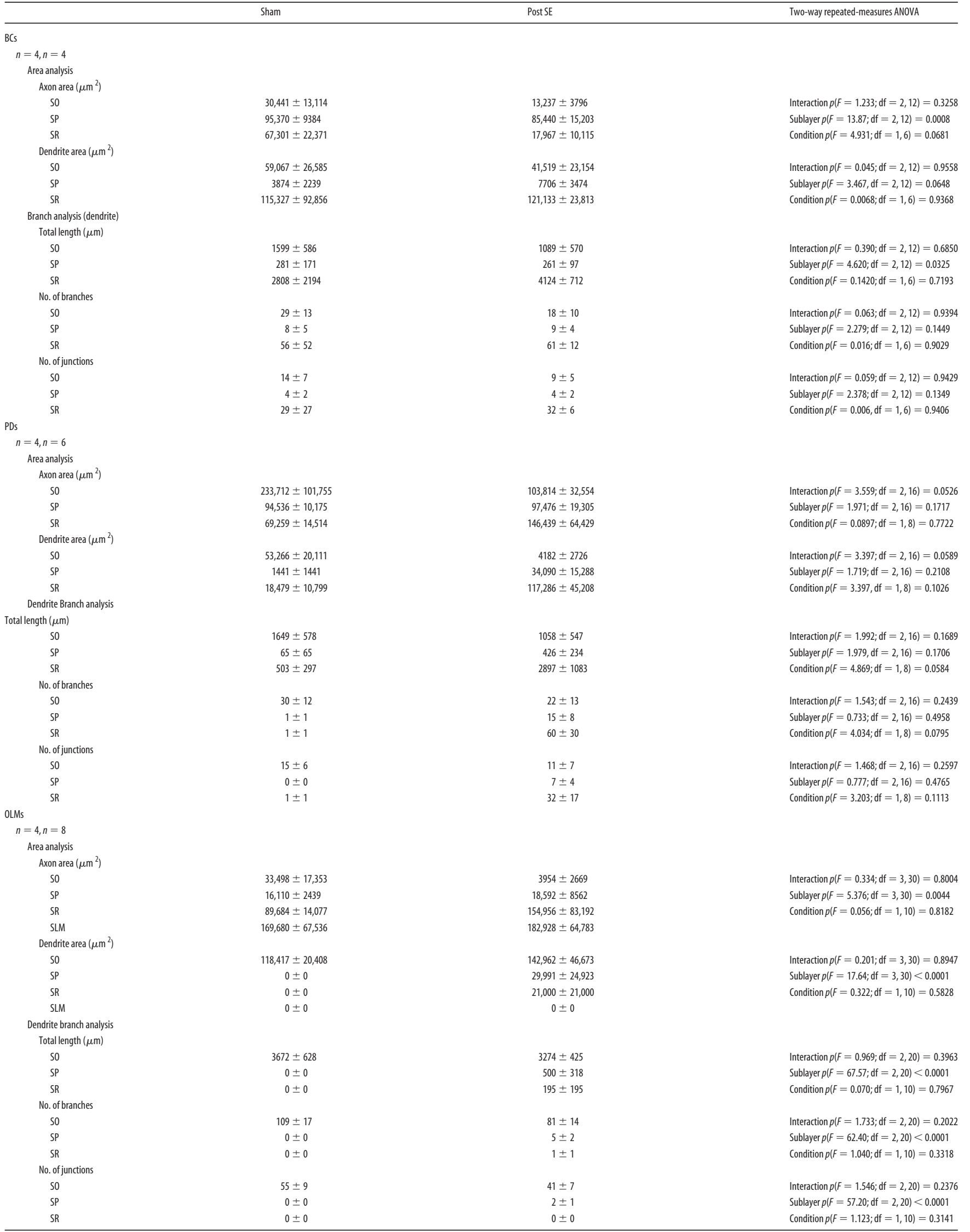

${ }^{a}$ The area covered by axon and dendrites of each interneuron type was quantified as the minimal surrounding polygon separated by hippocampal sublayer. SO, Stratum oriens; SP, stratum pyramidale; SR, stratum radiatum; SLM, Stratum lacunosum moleculare. Additionally, dendritic trees were reconstructed, and their total length, number of branches, and number of junctions were quantified separated by sublayer. An effect of condition (Sham or Post SE) and sublayer (SO, SP, SR, SLM) was examined using two-way repeated-measures ANOVA for each parameter investigated. 
Table 2. Bouton density ${ }^{a}$

\begin{tabular}{lllll}
\hline & & Sham & Post SE & Two-way ANOVA \\
\hline BCs & $n=4, n=4$ & $0.19 \pm 0.01$ & $0.18 \pm 0.03$ & Interaction $p(F=0.3028 ; \mathrm{df}=2,24)=0.7415$ \\
PDs & $n=4, n=6$ & $0.18 \pm 0.02$ & $0.20 \pm 0.01$ & Cell type $p(F=1.396 ; \mathrm{df}=2,24)=0.2670$ \\
OLMs & $n=4, n=8$ & $0.20 \pm 0.02$ & $0.22 \pm 0.02$ & Condition $p(F=0.3037 ; \mathrm{df}=1,24)=0.5866$
\end{tabular}

${ }^{a}$ The axonal bouton density was quantified as the mean number of boutons per micrometer on five random terminal branches. An effect of condition (Sham or Post SE) and cell type (BC, PD, OLM) was examined using ordinary two-way ANOVA.

In the three groups of interneurons, we then asked how they are recruited by feedback excitatory inputs from CA1 pyramidal neurons. Feedback excitation elicited with a stimulus electrode placed into the alveus revealed distinctive forms of short-term plasticity in the different types of CA1 interneurons in shamcontrol animals, as described previously (Pothmann et al., 2014) (Fig. $1 C, D$, left; $n=7, n=10$, and $n=8$ for BC, PD, and OLM, respectively; examples in Fig. $1 E$ ). Both basket and PD cells received a large amplitude excitatory input at the beginning of a 50 $\mathrm{Hz}$ stimulus train (10 stimulations) that was followed by synaptic depression (by $-47.3 \pm 11.5 \%$ and $-25.4 \pm 14.5 \%$, respectively; Fig. $1 D$, left; examples in Fig. $1 E$ ). In contrast, OLM interneurons showed strong facilitation (by $70.6 \pm 10.2 \%$ ). Next, we compared these cell-specific short-term dynamics to chronically epileptic animals (Fig. $1 D$, right; Fig. $1 E$, gray traces; $n=13, n=10$, and $n=14$ for BC, PD, and OLM cells, respectively). Basket and PD cells displayed no depression in EPSP size over the stimulus train (Figs. $1 D, 2: 0.1 \pm 11.8 \%$ and $0.7 \pm 15.9 \%$, respectively). OLM neurons maintained a strong facilitation (by $71.6 \pm 7.9 \%$ ). Significant differences between control and epileptic animals were observed only for BCs (Fig. 1F; BCs: $p_{(U=13 ; n=7, n=13)}=0.0084$; PDs: $p_{(U=32 ; n=10, n=10)}=0.1883$; OLMs: $p_{(U=52 ; n=8, n=14)}=$ 0.8776; Mann-Whitney $U$ test).

During exploratory behavior and REM sleep, firing of hippocampal pyramidal cells is phase-locked to the theta rhythm, a field potential oscillation of $5-10 \mathrm{~Hz}$ (Ylinen et al., 1995). We therefore explored the excitation of the different types of interneurons within feedback circuits with a theta burst protocol consisting of a high-frequency component (three stimuli at 100 $\mathrm{Hz}$ ) repeated 10 times at a frequency of $5 \mathrm{~Hz}$ (Fig. $2 A$ ). The peak response obtained during theta patterned bursts decreased strongly during the train in both BCs and PD cells of control animals (by $-34.6 \pm 6.1 \%$ and $-33.8 \pm 11.5 \%$, respectively, $n=$ 7 and $n=11)$, but increased in OLM neurons $(38.6 \pm 11.3 \%, n=$ 8; Fig. $2 B$ ). In epileptic animals, both $\mathrm{BCs}$ and $\mathrm{PD}$ cells displayed a loss of depression during the theta stimulation train (Fig. $2 B, C$, gray traces; change by $-8.7 \pm 8.7$ and $10.7 \pm 11.9$, respectively). This significantly differed from sham control animals (BCs: $p_{(U=16 ; n=7, n=14)}=0.0123$; PDs: $p_{(U=19 ; n=11, n=10)}=0.0101$; Mann-Whitney $U$ test). In OLM cells, the EPSP showed similar dynamics (Fig. $2 B$, gray traces; change by $54.4 \pm 9.1 \%, n=14$; OLMs: $p_{(U=38 ; n=8, n=14)}=0.2308$, Mann-Whitney $U$ test compared with sham-control animals).

\section{Altered paired-pulse facilitation at feedback inputs onto different interneuron types}

Altered dynamics of recruitment of CA1 BCs and PD interneurons might be due to reduced basal release probability of feedback excitatory synapses (Fig. 3). We therefore examined whether paired pulse facilitation, a commonly used indicator of release probability (Pouille and Scanziani, 2004), is altered in epileptic animals at excitatory feedback synapses. Indeed, in BCs, the paired pulse ratio was significantly altered from $0.61 \pm 0.07$ in control animals to $0.97 \pm 0.10$ in epileptic animals (Fig. $3 A, B$, left; $p_{(U=9 ; n=9, n=10)}=0.0021$, Mann-Whitney $U$ test). In PD cells, the paired pulse ratio was not significantly altered (Fig. $3 A, B$, middle; $0.76 \pm 0.13$ vs $0.95 \pm 0.17, p_{(U=35 ; n=10, n=9)}=$ 0.4404 , Mann-Whitney $U$ test). OLM neurons showed strong paired-pulse facilitation in both control and epileptic animals and no significant differences in the paired pulse ratio could be observed (Fig. $3 A$, $B$, right; PPR of $1.75 \pm 0.14, n=11$ and PPR of $1.74 \pm 0.35, n=12$ for sham and epileptic animals, respectively; $p_{(U=66 ; n=11, n=12)}>0.9999$, Mann-Whitney $U$ test $)$. Thus, the robust finding of altered recruitment of BCs in feedback circuits using different stimulation protocols could be due to altered release probability.

\section{Altered active and passive properties of interneurons}

In addition to the dynamics of the synaptic excitatory drive, the recruitment of interneurons within inhibitory networks also depends strongly on their passive and active properties. We found profound changes in the passive properties of interneurons in chronic epilepsy. In BCs and OLM cells, the input resistance was strongly reduced (Table 3; $p_{(t=2.696, \mathrm{df}=22)}=0.0132$ and $p_{(t=4.327, \mathrm{df}=24)}=0.0002$ for BCs and OLM cells, respectively, Student's $t$ test). In pyramidal neurons, the input resistance was also reduced in epileptic animals $\left(p_{(t=2.189, \mathrm{df}=25)}=0.0382\right.$, Student's $t$ test), albeit to a lower degree than in interneurons. The active properties of all three classes of interneurons under investigation were unchanged, whereas pyramidal neurons showed a more depolarized AP threshold (Table 3; $p_{(t=2.530, \mathrm{df}=25)}=$ 0.0181 , Student's $t$ test) and a reduced AP half-width in epileptic animals (see Table 3; $p_{(t=4.239, \mathrm{df}=25)}=0.0003$, Student's $t$ test).

We then examined the firing behavior induced by increasing $1 \mathrm{~s}$ current injections in sham-control and epileptic animals for each of these cell categories (Fig. 4; BCs: $n=9, n=6$; PDs: $n=10$, $n=7$; OLMs: $n=9, n=9$; for sham and epileptic animals, respectively). The maximally obtained firing rate (with up to 800 pA current injections) was significantly increased only in pyramidal neurons, but not interneurons (Fig. $4 C$; $n=13, n=14$; $22.2 \pm 1.2$ vs $29.4 \pm 1.9 \mathrm{~Hz}$ for sham and epileptic animals, respectively; $p_{(t=2.940, \mathrm{df}=25)}=0.0070$; Student's $t$ test). We then determined the current injection needed to achieve halfmaximal discharge rates through sigmoidal fits to the individual cells (Fig. $4 D$; black lines in Fig. $4 B$ are fits to the average data). Consistent with the large reduction in input resistance, we found a pronounced shift of the input-output relation in both BCs and OLM interneurons (Fig. $4 B, D$; BCs: $316 \pm 55$ vs $519 \pm 55 \mathrm{pA}$ for sham and epileptic animals, respectively, $p_{(t=2.500, \mathrm{df}=13)}=0.0266$; OLM cells: $186 \pm 20 \mathrm{vs} 343 \pm 36$ $\mathrm{pA}, p_{(t=3.924, \mathrm{df}=17)}=0.0011$; Student's $t$ tests). A similar change was seen in pyramidal neurons (Fig. 4D; $259 \pm 19$ vs $382 \pm 26 \mathrm{pA}$ for sham and epileptic animals, respectively, $p_{(t=3.753, \mathrm{df}=25)}=0.0009$, Student's $t$ test). In PD neurons, the effect was not statistically significant $(341 \pm 54$ vs $484 \pm 58 \mathrm{pA}$ for sham and epileptic animals, respectively, $p_{(t=1.778, \mathrm{df}=15)}=$ 0.0956 , Student's $t$ test). We additionally quantified the rheobase currents and found a significant rightward shift across all cell types (BCs: $136 \pm 24$ vs $238 \pm 42, p_{(t=2.275, \mathrm{df}=13)}=0.0405$; PDs: $150 \pm 38$ vs $293 \pm 47, p_{(t=2.386, d f=15)}=0.0307$; OLMs: $65 \pm 11$ vs $175 \pm 27, p_{(t=3.822, \mathrm{df}=16)}=0.0015$; PCs: $158 \pm 15$ vs $234 \pm 26, p_{(t=2.482, \mathrm{df}=25)}=0.0201$; each for sham and epileptic animals, respectively, Student's $t$ test). Thus, in addition to changes in the properties of excitatory synapses driving them, there are large differences in intrinsic properties that reduce the ability of CA1 interneurons to be recruited synap- 
A

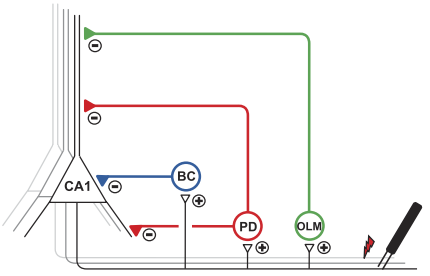

Theta burst protocol

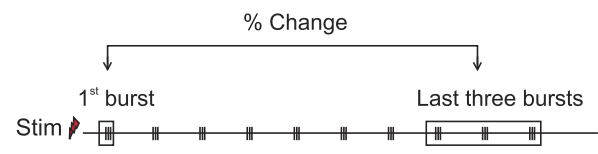

B
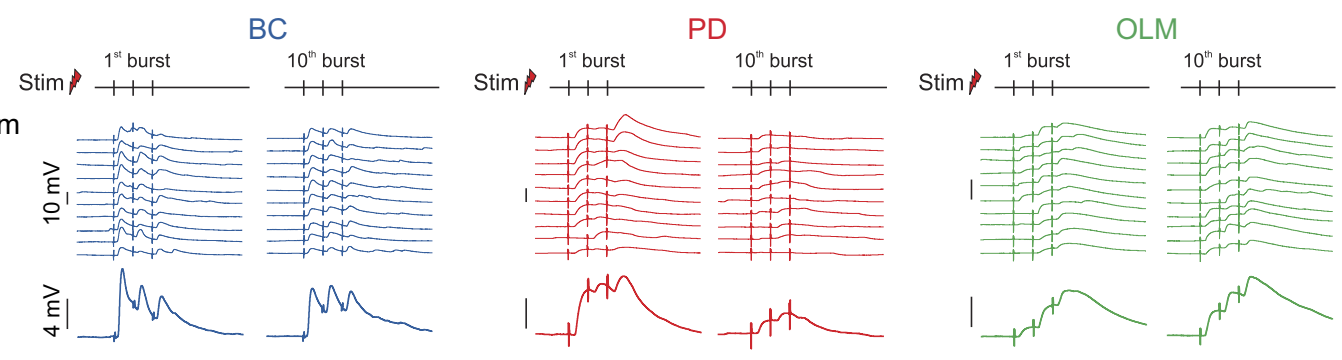

Sham

post SE
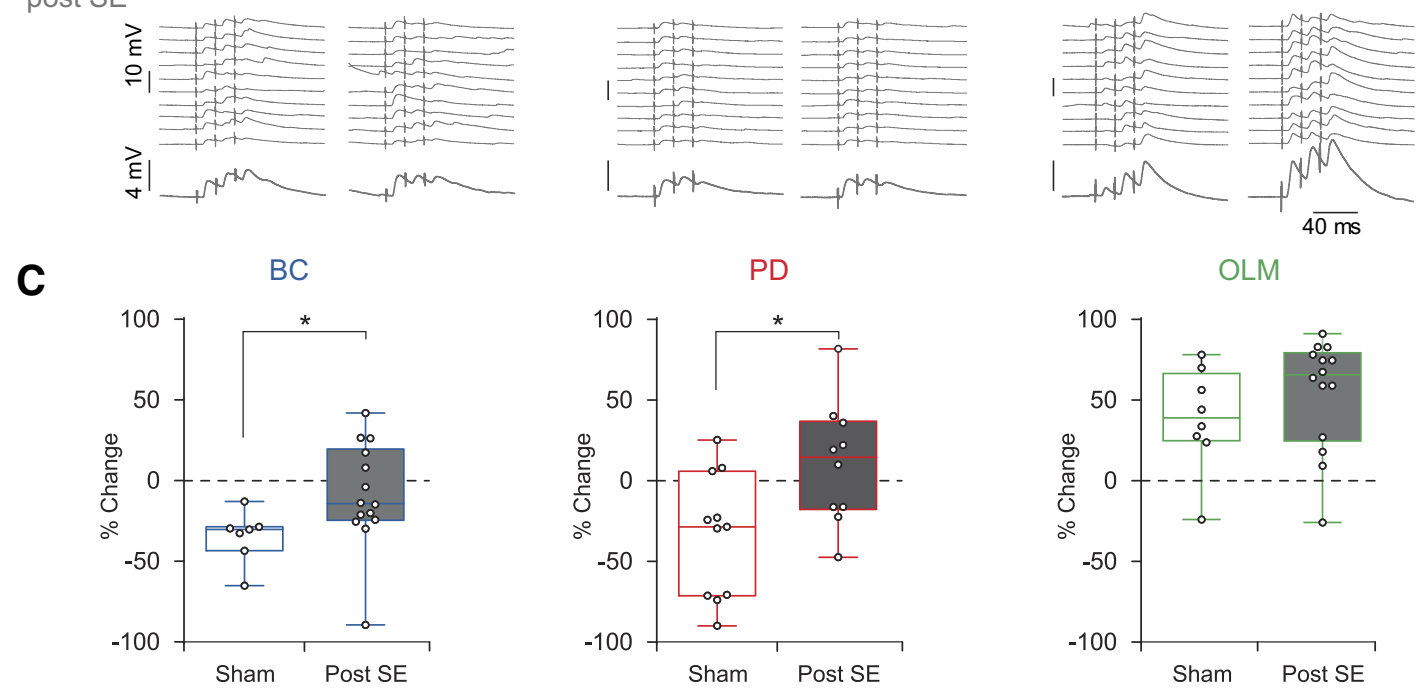

Figure 2. Epilepsy-associated changes in feedback recruitment during theta patterned burst stimulation. $\boldsymbol{A}$, Schematic of the recording configuration and the theta burst protocol. $\boldsymbol{B}$, Representative recordings of a $\mathrm{BC}$, a proximal dendritic interneuron (PD), and an $0 \mathrm{LM}$ cell during theta burst stimulation. The stimulation train consisted of three stimuli at $100 \mathrm{~Hz}$, repeated 10 times at a frequency of $5 \mathrm{~Hz}$. Only responses to the first and the 10th burst in the stimulus train are shown. For each condition and cell type, 10 single sweeps followed by their mean is shown. C, Quantification of the percentage change of the peak EPSP from the first to the last burst stimulation within the stimulation train. Both PD and BCs showed a significant reduction of synaptic depression. ${ }^{*} p=0.01$ for both cases (Mann-Whitney $U$ test).

tically. Furthermore, pyramidal cells in epileptic animals can in principle reach higher activity levels.

\section{Altered recruitment of feedback inhibition onto pyramidal cells in chronic epilepsy}

How do these changes collectively impact the time course and magnitude of feedback inhibition onto CA1 pyramidal cells? We directly examined this question by recording feedback inhibition elicited by alveus stimulation in control and epileptic animals in CA1 pyramidal neurons (Fig. 5A). During $50 \mathrm{~Hz}$ stimulation, the amplitude of IPSCs in control animals decreased strongly from the first to the 10th stimulus, with an average decrease of $-77.8 \pm$ $1.7 \%$ (Fig. $5 B, D$, open bar; $n=25$ ). In pilocarpine-treated animals, the depression in amplitude was significantly diminished (average decrease: $-44.9 \pm 7.6 \%, n=15$, Fig. $5 C, D$, gray bar; $p_{(U=56 ; n=25, n=15)}=0.0001$, Mann-Whitney $U$ test $)$.

We next compared the absolute amplitude of the IPSCs elicited at the beginning and the end of the stimulus trains in pilocarpine-treated and control animals (Fig. 5E). We observed a significant effect of stimulus time point, treatment, as well as an interaction (two-way repeated-measures ANOVA; time point: $p_{(F=51.28 ; \mathrm{df}=1,38)}<0.0001$; treatment: $p_{(F=7.42 ; \mathrm{df}=1,38)}=0.0097$; interaction: $\left.p_{(F=16.51 ; \mathrm{df}=1,38)}=0.0002\right)$. In control animals, the first IPSC had an average amplitude of $197.5 \pm 23.7 \mathrm{pA}$ (Fig. 5E), whereas in epileptic animals, IPSC amplitude was significantly smaller $\left(85.0 \pm 17.1 \mathrm{pA}, p_{(t=4.583, \mathrm{df}=76)}<0.0001\right.$, Bonferroni's multiple-comparisons post test). In contrast, the amplitude of the 10th IPSC in the stimulus train was not significantly changed in epileptic animals $\left(p_{(t=0.161, \mathrm{df}=76)}>0.9999\right.$, Bonferroni's multiple-comparisons post test). To further assess whether the changes above lead to an overall reduction in inhibitory input, the charge transfer over the whole stimulus train was analyzed (Fig. $5 F$ ), revealing a significant reduction in epileptic animals $(11.3 \pm 1.5 \mathrm{nA} \times \mathrm{ms}$ compared with $7.3 \pm 1.2 \mathrm{nA} \times \mathrm{ms}$ for control and epileptic animals, respectively. $p_{(t=2.066, \mathrm{df}=42)}=$ 0.0450 , Student's $t$ test).

During theta patterned feedback stimulation, the differences in feedback inhibitory dynamics between control and epileptic animals were even more pronounced than during $50 \mathrm{~Hz}$ stimulation. In control animals, the IPSC amplitude decreased by $-51.44 \pm 3.81 \%$ 
A

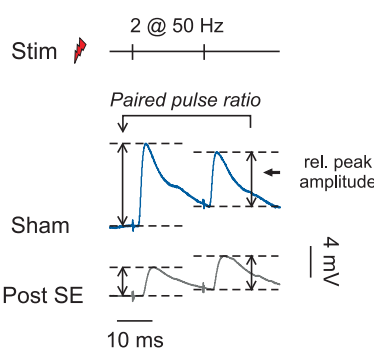

B

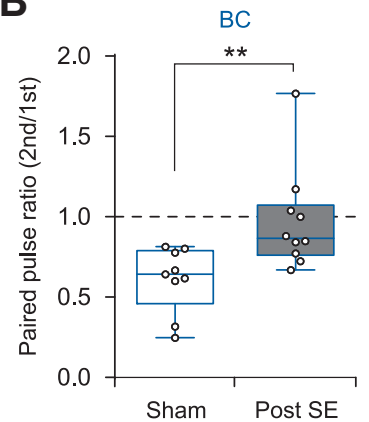

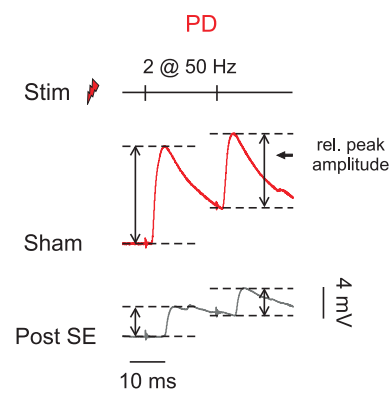

PD

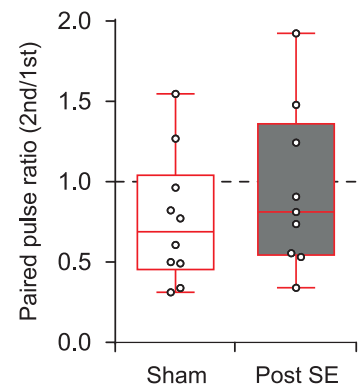

OLM

Stim $-2 @ 50 \mathrm{~Hz}$

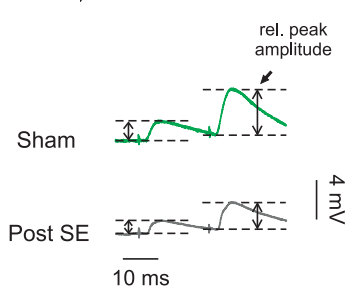

OLM

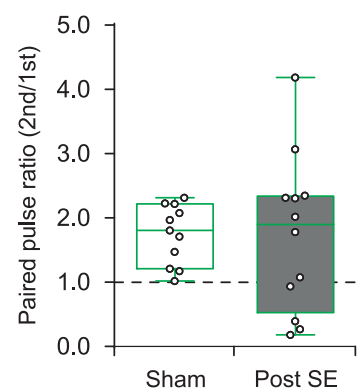

Figure 3. Altered paired-pulse ratios at feedback inputs onto different interneuron types. $A$, Representative recordings of $B C S$, PD cells, and $0 L M$ cells in sham control (top, blue, red, and green, respectively) and epileptic animals (Post SE, bottom, gray traces). $\boldsymbol{B}$, Paired-pulse ratio calculated as the ratio of the second to the first EPSP peak amplitudes. Data taken from $50 \mathrm{~Hz}$ stimulation protocols depicted in Figure 1. Relative peak amplitude was measured as indicated by the dashed lines in $\boldsymbol{A}$. BCs: $p=0.002$ (Mann-Whitney $U$ test). ${ }^{* *} p<0.01$.

Table 3. Active and passive membrane properties in the four cell types studied, and their changes in chronic epilepsy ${ }^{a}$

\begin{tabular}{|c|c|c|c|c|}
\hline & & Sham & Post SE & $t$ test \\
\hline \multicolumn{5}{|l|}{$\mathrm{BCs}$} \\
\hline AP amplitude (mV) & $n=10,14$ & $74.18 \pm 3.38$ & $72.40 \pm 2.21$ & $p(t=0.458, \mathrm{df}=22)=0.6510$ \\
\hline Maximum slope (mV/ms) & $n=10,14$ & $412.60 \pm 38.06$ & $496.18 \pm 39.10$ & $p(t=1.481, \mathrm{df}=22)=0.1527$ \\
\hline Threshold (mV) & $n=10,14$ & $-42.85 \pm 0.83$ & $-44.48 \pm 0.73$ & $p(t=1.456, \mathrm{df}=22)=0.1595$ \\
\hline AP half-width (ms) & $n=10,14$ & $0.46 \pm 0.08$ & $0.34 \pm 0.03$ & $p(t=1.493, \mathrm{df}=22)=0.1496$ \\
\hline $\mathrm{R}_{\text {in }}(m \Omega)$ & $n=10,14$ & $170.76 \pm 34.23$ & $80.12 \pm 14.75$ & $p(t=2.696, \mathrm{df}=22)=0.0132$ \\
\hline$C(\mathrm{nF})$ & $n=10,14$ & $0.10 \pm 0.01$ & $0.15 \pm 0.02$ & $p(t=2.348, \mathrm{df}=22)=0.0283$ \\
\hline $\operatorname{RMP}(\mathrm{mV})$ & $n=10,14$ & $-51.78 \pm 1.96$ & $-52.38 \pm 1.10$ & $p(t=0.291, \mathrm{df}=20)=0.7741$ \\
\hline Sag (mV) & $n=9,11$ & $-2.10 \pm 0.60$ & $-2.17 \pm 0.31$ & $p(t=0.120, \mathrm{df}=18)=0.9062$ \\
\hline \multicolumn{5}{|l|}{ PD cells } \\
\hline AP amplitude (mV) & $n=13,9$ & $71.17 \pm 2.78$ & $64.51 \pm 3.34$ & $p(t=1.531, \mathrm{df}=20)=0.1415$ \\
\hline Maximum slope (mV/ms) & $n=13,9$ & $429.95 \pm 31.97$ & $457.42 \pm 58.86$ & $p(t=0.443, \mathrm{df}=20)=0.6624$ \\
\hline Threshold (mV) & $n=13,9$ & $-42.95 \pm 0.89$ & $-40.69 \pm 2.05$ & $p(t=1.130, \mathrm{df}=20)=0.2720$ \\
\hline AP half-width (ms) & $n=13,9$ & $0.37 \pm 0.04$ & $0.38 \pm 0.09$ & $p(t=0.060, \mathrm{df}=20)=0.9529$ \\
\hline $\mathrm{R}_{\mathrm{in}}(\mathrm{M} \Omega)$ & $n=13,9$ & $172.09 \pm 38.64$ & $107.66 \pm 45.64$ & $p(t=1.074, \mathrm{df}=20)=0.2967$ \\
\hline$C(\mathrm{nF})$ & $n=13,9$ & $0.10 \pm 0.01$ & $0.16 \pm 0.03$ & $p(t=2.101, \mathrm{df}=20)=0.0485$ \\
\hline $\mathrm{RMP}(\mathrm{mV})$ & $n=13,9$ & $-51.77 \pm 1.66$ & $-54.44 \pm 1.89$ & $p(t=1.051, \mathrm{df}=20)=0.3056$ \\
\hline Sag (mV) & $n=12,7$ & $-1.63 \pm 0.33$ & $-1.55 \pm 0.14$ & $p(t=0.184, d f=17)=0.8563$ \\
\hline \multicolumn{5}{|l|}{ OLM cells } \\
\hline AP amplitude (mV) & $n=11,14$ & $75.04 \pm 2.51$ & $76.86 \pm 2.42$ & $p(t=0.517, \mathrm{df}=23)=0.6104$ \\
\hline Maximum slope (mV/ms) & $n=11,14$ & $350.95 \pm 34.89$ & $388.12 \pm 19.83$ & $p(t=0.976, \mathrm{df}=23)=0.3392$ \\
\hline Threshold (mV) & $n=11,14$ & $-41.73 \pm 0.85$ & $-42.95 \pm 0.68$ & $p(t=1.129, \mathrm{df}=23)=0.2705$ \\
\hline AP half-width (ms) & $n=11,14$ & $0.51 \pm 0.04$ & $0.46 \pm 0.03$ & $p(t=1.101, \mathrm{df}=23)=0.2824$ \\
\hline$R_{\text {in }}(m \Omega)$ & $n=12,14$ & $181.27 \pm 28.68$ & $63.19 \pm 6.18$ & $p(t=4.327, \mathrm{df}=24)=0.0002$ \\
\hline$C(\mathrm{nF})$ & $n=12,14$ & $0.14 \pm 0.02$ & $0.19 \pm 0.02$ & $p(t=2.179, \mathrm{df}=24)=0.0394$ \\
\hline $\mathrm{RMP}(\mathrm{mV})$ & $n=11,14$ & $-52.36 \pm 1.42$ & $-50.21 \pm 1.16$ & $p(t=1.187, \mathrm{df}=23)=0.2475$ \\
\hline Sag $(m V)$ & $n=11,14$ & $-4.34 \pm 0.48$ & $-5.56 \pm 0.72$ & $p(t=1.384, \mathrm{df}=23)=0.1796$ \\
\hline \multicolumn{5}{|l|}{ Pyramidal cells } \\
\hline AP amplitude (mV) & $n=13,14$ & $86.41 \pm 1.43$ & $82.48 \pm 1.57$ & $p(t=1.841, \mathrm{df}=25)=0.0775$ \\
\hline Maximum slope (mV/ms) & $n=13,14$ & $390.62 \pm 12.08$ & $411.33 \pm 16.60$ & $p(t=0.996, \mathrm{df}=25)=0.3289$ \\
\hline Threshold (mV) & $n=13,14$ & $-45.36 \pm 0.83$ & $-41.64 \pm 1.19$ & $p(t=2.530, \mathrm{df}=25)=0.0181$ \\
\hline AP half-width (ms) & $n=13,14$ & $0.91 \pm 0.02$ & $0.81 \pm 0.02$ & $p(t=4.239, \mathrm{df}=25)=0.0003$ \\
\hline $\mathrm{R}_{\text {in }}(\mathrm{M} \Omega)$ & $n=13,14$ & $63.65 \pm 5.53$ & $45.18 \pm 6.30$ & $p(t=2.198, \mathrm{df}=25)=0.0382$ \\
\hline $\mathrm{C}(\mathrm{nF})$ & $n=13,14$ & $0.35 \pm 0.03$ & $0.39 \pm-0.03$ & $p(t=1.056, \mathrm{df}=25)=0.3009$ \\
\hline $\mathrm{RMP}(\mathrm{mV})$ & $n=13,14$ & $-56.85 \pm 1.31$ & $55.07 \pm 0.87$ & $p(t=1.145, \mathrm{df}=25)=0.2629$ \\
\hline Sag (mV) & $n=12,14$ & $-3.18 \pm 0.29$ & $-4.30 \pm 0.19$ & $p(t=3.298, \mathrm{df}=24)=0.0030$ \\
\hline
\end{tabular}

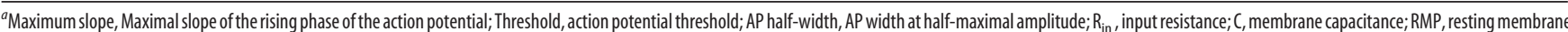
potential; Sag, hyperpolarizing sag of the membrane potential caused by the presence of $h$-current. 
A
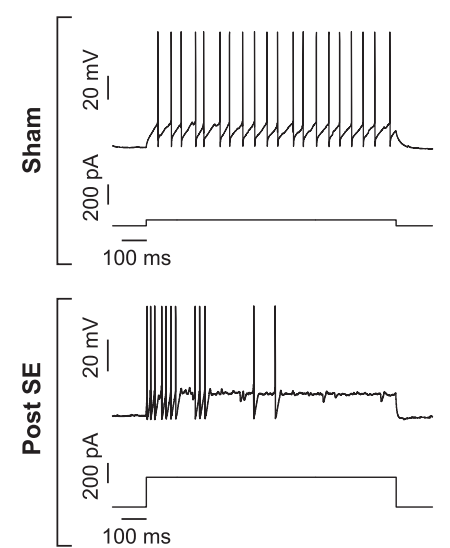

B

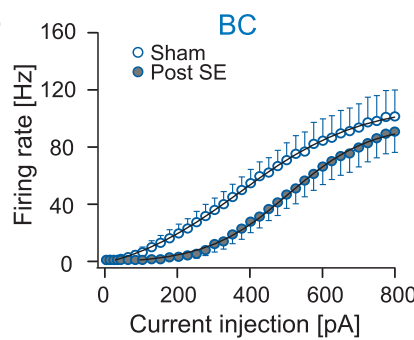

C

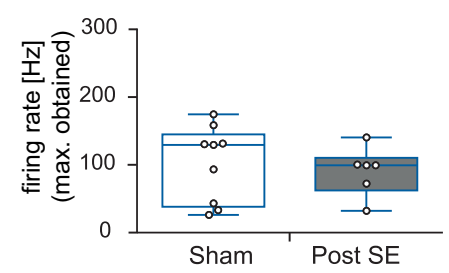

D

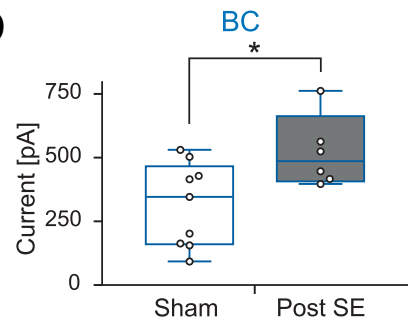

PD
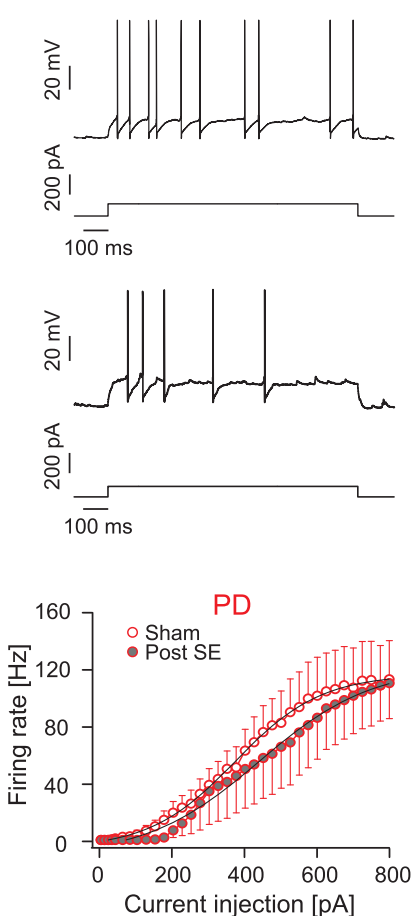

PD
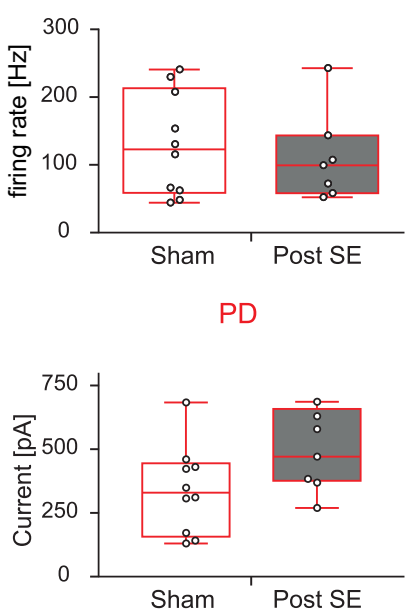

OLM
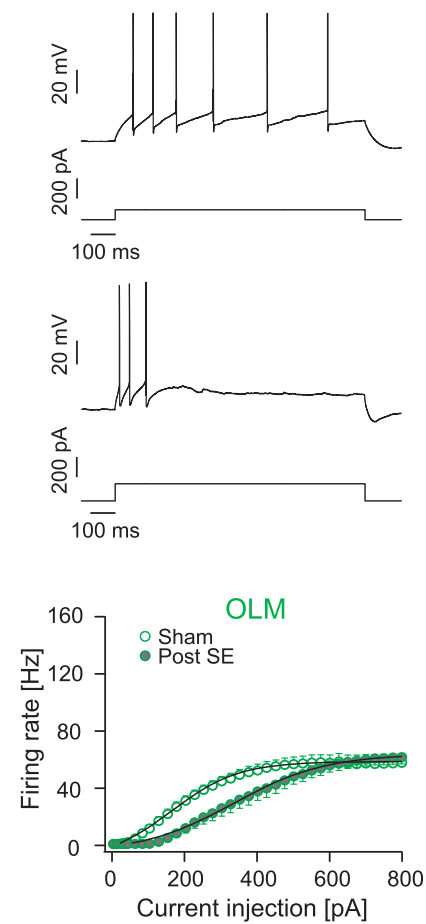

OLM
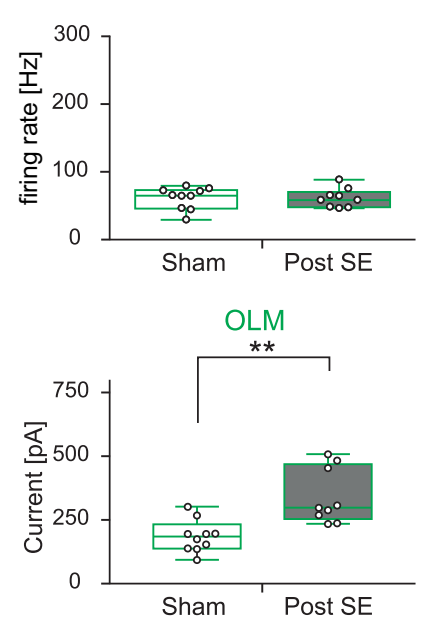

PC
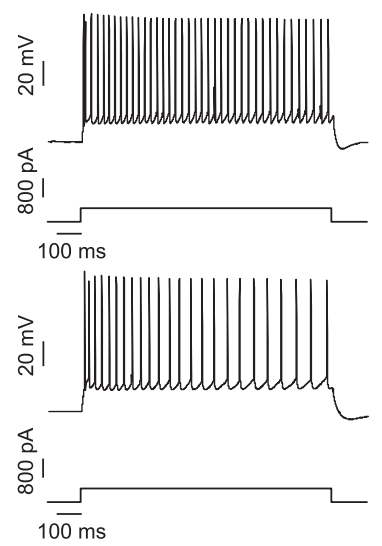

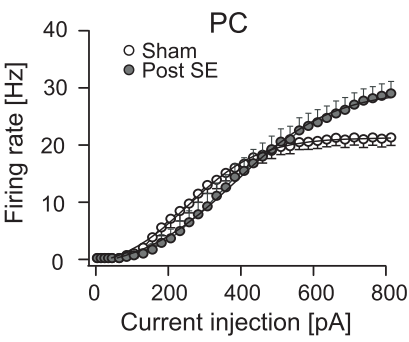

$\mathrm{PC}$
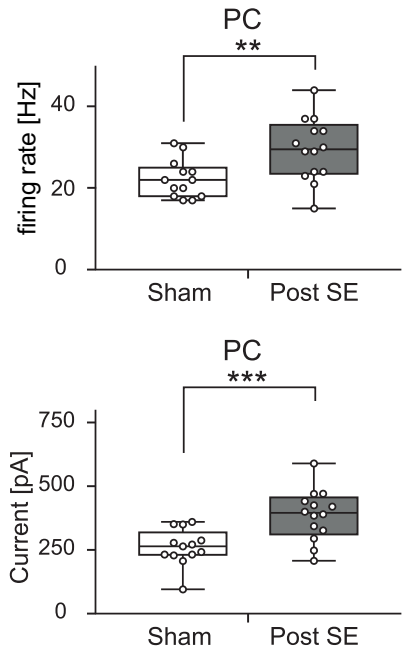

Figure 4. Excitability of interneurons in epileptic animals is altered. $\boldsymbol{A}$, Representative discharge responses of the basket, $\mathrm{PD}$, and $0 \mathrm{LM}$ neurons depicted in $\boldsymbol{A}, \boldsymbol{B}$ to $1 \mathrm{~s}$ current injections. $\boldsymbol{B}$, Input-output relation of basket, $\mathrm{PD}, 0 \mathrm{LM}$, and pyramidal cells. The average firing rate was calculated and plotted versus the current injection. Solid line indicates sigmoid fit. $C$, Determination of the maximally obtained discharge rate during the $1 \mathrm{~s}$ current injections (with up to $800 \mathrm{pA}$ injections). PCs: $p=0.007$, Student's $t$ test. $\boldsymbol{D}$, Analysis of the current injection required to attain a half-maximal firing rate as determined by sigmoid fits of input-output curves of individual cells. $p=0.02, p=0.001$, and $p=0.0009$ for BCs, $0 \mathrm{LMs}$, and PCs, respectively (Student's $t$ test). ${ }^{*} p<$ $0.05,{ }^{* *} p<0.01,{ }^{* * *} p<0.001$.

from the first to the 10th burst (Fig. $6 A, C ; n=9$ ). In epileptic animals, this phenomenon was strongly attenuated, with an average reduction of peak IPSC amplitude of only $-10.16 \pm 11.01 \%$ (Fig. $6 B, C ; p_{(U=9 ; n=9, n=9)}=0.0040$, Mann-Whitney $U$ test $)$. As for the $50 \mathrm{~Hz}$ stimulation trains, epileptic animals displayed a strong reduction of IPSC amplitudes at the first burst stimulation in the train, but not the last (Fig. 6D; two-way repeatedmeasures ANOVA; stimulus time point: $p_{(F=30.67, \mathrm{df}=1,16)}<$ 0.0001; treatment: $\left.p_{(F=8.36 ;} \mathrm{df}=1,16\right)=0.0106$; interaction: $p_{(F=16.70 ; \mathrm{df}=1,16)}=0.0009$; Bonferroni's multiple-comparison post test of control vs treatment: $p_{(t=6.806, \mathrm{df}=16)}<0.0001$ and $p_{(t=1.027, \mathrm{df}=16)}=0.6395$ for first and 10th stimulus, respectively). Thus, net inhibition of the feedback circuit shows markedly changed dynamics.
Generation of a computational model of the feedback circuit Generation of a simple population model allowed us to further interpret our data. The changes between the response properties of neuron populations in sham-control and epileptic animals were reflected by quantitative and qualitative changes of model parameters. We focused on inhibitory BC populations because we expected them to be most influential for controlling spike output of CA1 neurons. Indeed, BCs (1) provide the dominant perisomatic inhibition to pyramidal neurons and (2) showed the most robust changes in feedback activation and intrinsic properties in chronic epilepsy (see Figs. 1-4).

The pyramidal neuron and $\mathrm{BC}$ population activities are represented by two population rates, $\mathrm{r}_{\mathrm{PY}}(\mathrm{t})$ and $\mathrm{r}_{\mathrm{BC}}(\mathrm{t})$, respectively (see Fig. 8A; see Materials and Methods). We first modeled the 

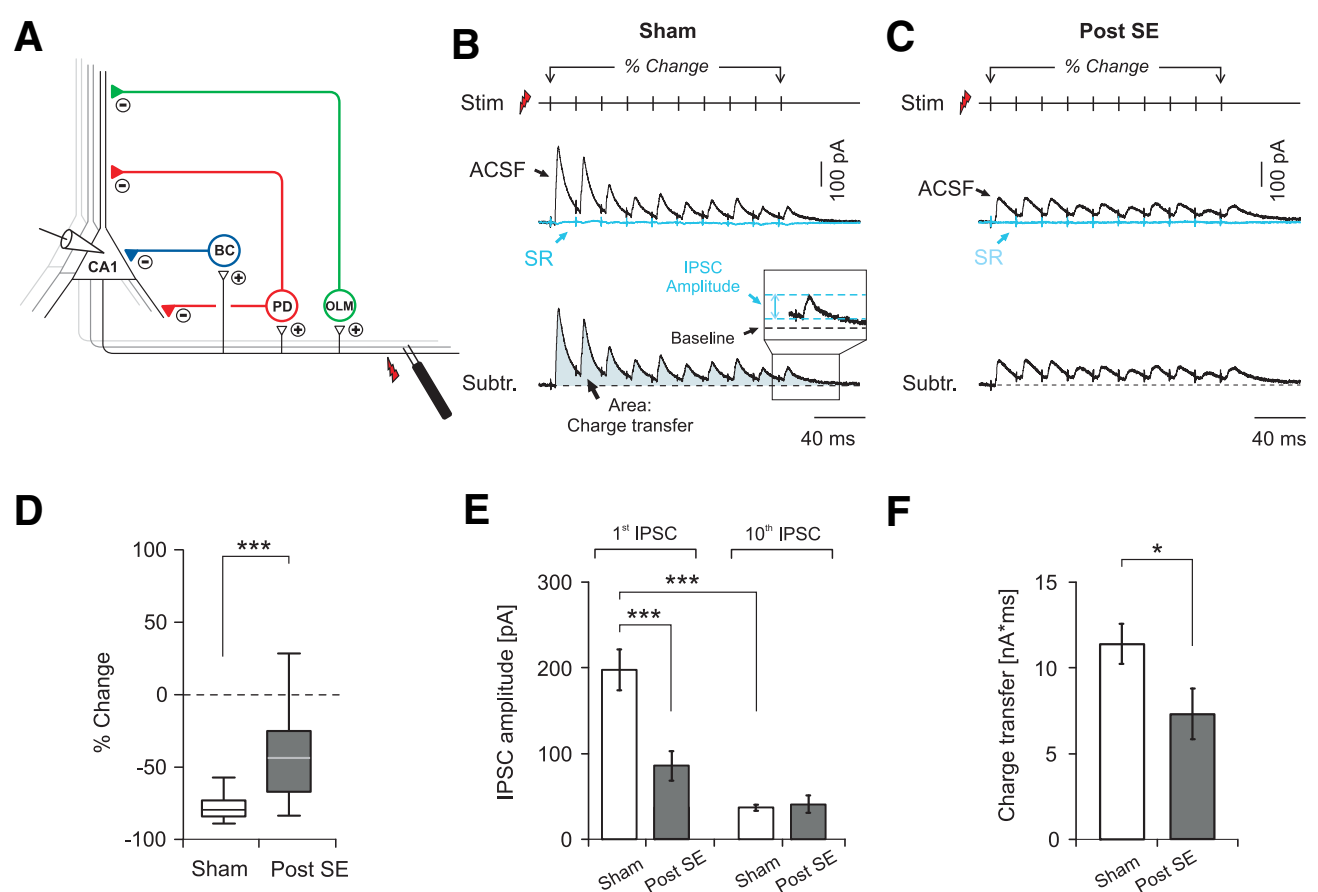

Figure 5. Changes in feedback inhibition onto CA1 pyramidal cells in epileptic animals. $A$, Schematic of the recording configuration. $\boldsymbol{B}$, Top, Representative recordings of feedback IPSCS elicited by a train of 10 stimuli at $50 \mathrm{~Hz}$ in a sham-injected animal before (ACSF) and after application of gabazine (SR, light blue trace). Bottom, The gabazine-sensitive component isolated by subtraction. Blue shading represents the area corresponding to the charge transfer. C, Equivalent representative recordings in an epileptic animal. D, Percent change in IPSC peak amplitude from the first to the 10th stimulus. IPSC amplitude was measured as indicated in $\boldsymbol{A}$ (dashed blue lines). ${ }^{* * *} p=0.0002$ (Mann-Whitney $\boldsymbol{U}$ test). $\boldsymbol{E}$, Comparison of absolute IPSC amplitude between control and epileptic animals for the first and 10th IPSC. ${ }^{* *} p<0.0001$ in both cases (Bonferroni post tests). $\boldsymbol{F}$, Quantification of the charge transfer calculated over the whole stimulus train, as indicated in $\boldsymbol{B}$ (blue area). ${ }^{*} p=0.04$ (Student's $t$ test).

feedback excitatory synapse activating $\mathrm{BCs}$ and the average $\mathrm{BC}$ population response to excitatory stimulation using a current-based synapse that exhibits STP and a simple leaky integrator model of the BC population. Short-term depression is modeled as depletion of a continuously replenishing pool of vesicles, where the "asymptotic release fraction" corresponds to the release fraction of vesicles assuming an infinitely long preceding interspike interval and the "timescale" measures how long replenishment takes. We determined the values of the model parameters that best capture the dynamics of activation of BCs by fitting the model to the average of the voltage traces measured in response to the stimulation of feedback excitatory synapses (as shown in Figs. 1, 2). This provided an accurate description of the excitatory drive to the population of BCs (see Materials and Methods).

The model allowed a good fit to the experimental data both in control animals and epileptic animals (Fig. 7A, left vs right; $R^{2}=0.98$ for both control and epileptic animals) and yields quantitative estimates for the effective characteristics of the BC population, such as the asymptotic release fraction and the timescale of replenishment. To verify that the fitting procedure was not trapped in a local min-
A

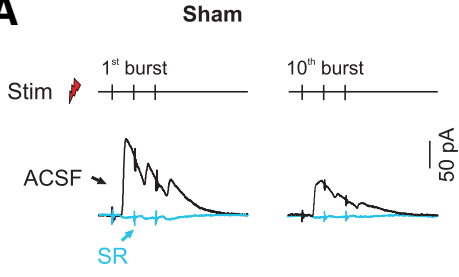

B
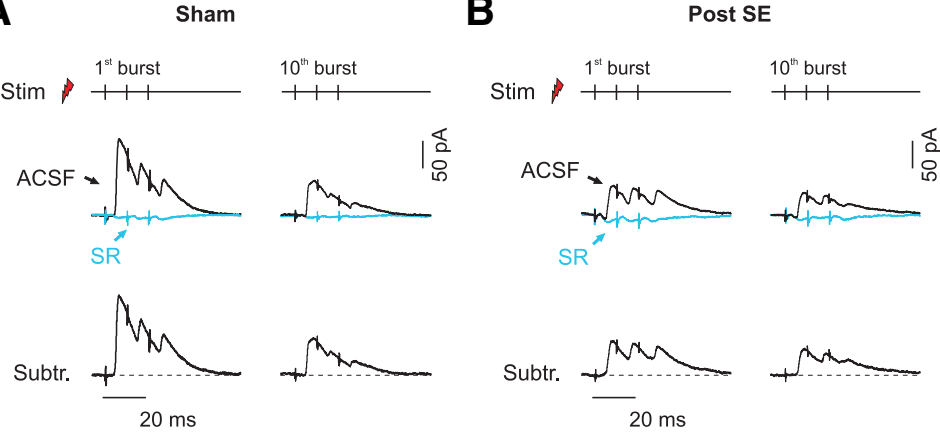

C

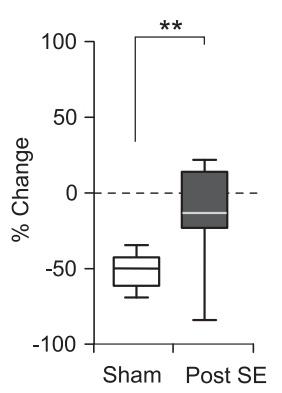

D

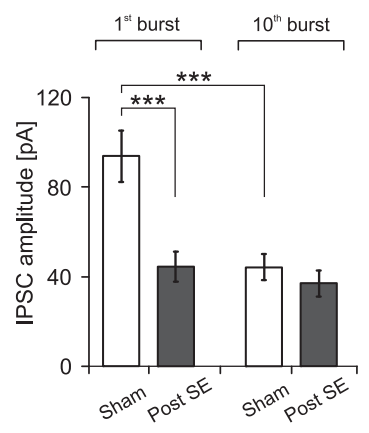

Figure 6. Changes in inhibitory input onto CA1 neurons during theta patterned feedback activity. A, Top, Representative recordings of feedback IPSCs in a sham-injected animal before (ACSF) and after application of gabazine (SR, light blue). Bottom, The gabazine-sensitive component isolated by subtraction. Only the response to the first and the 10th burst stimulation are shown. $B$, Representative recordings in an epileptic animal, analogous to $A$. C, Percent change in IPSC amplitude over the burst train. The first IPSC of the first burst was compared with the first IPSC of the last three bursts. IPSC amplitudes were measured as indicated in $\boldsymbol{A}$ (dashed blue lines). ${ }^{* *} p=0.002$ (Mann-Whitney U test). $\boldsymbol{D}$, Comparison of peak IPSC amplitude between control and epileptic animals for the first and 10th burst. ${ }^{* * *} p<0.001$. 

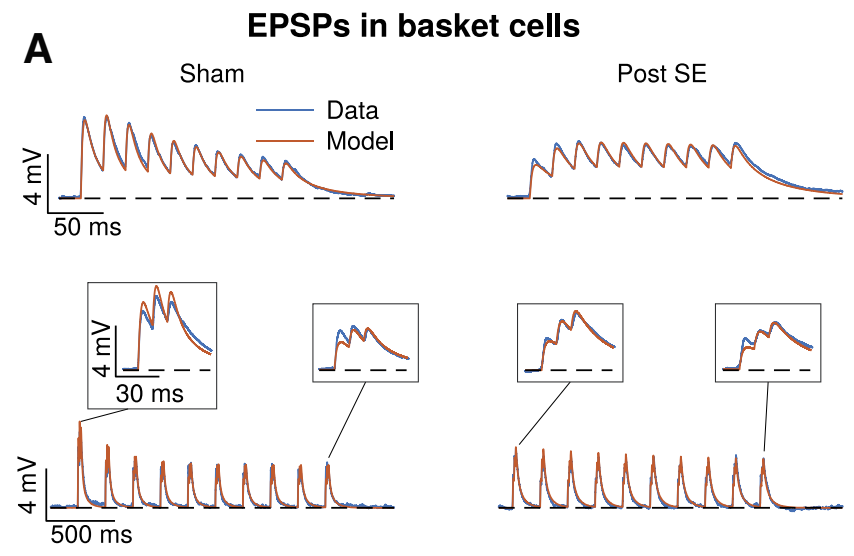

C

Short-term depression
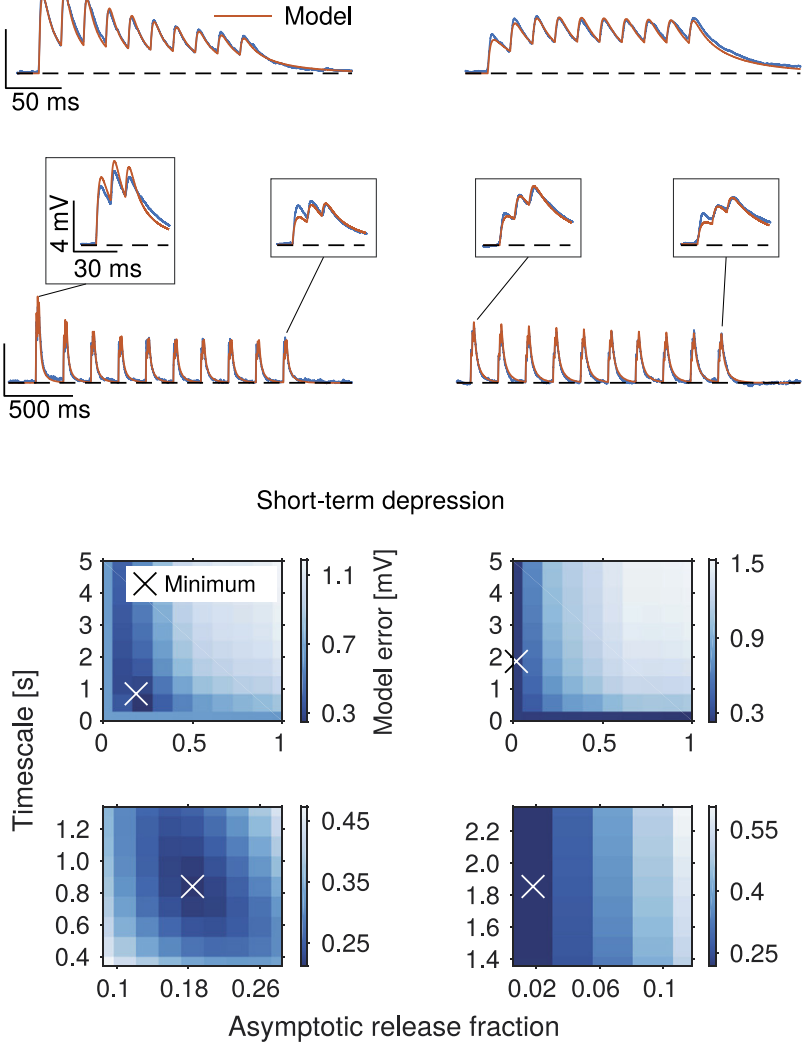

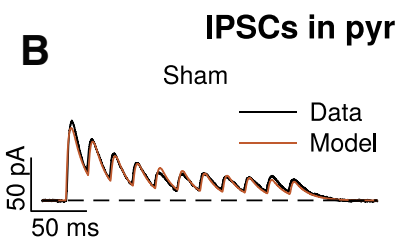

Post SE
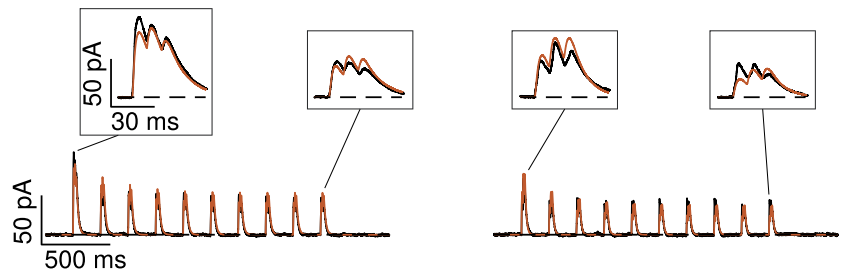

D

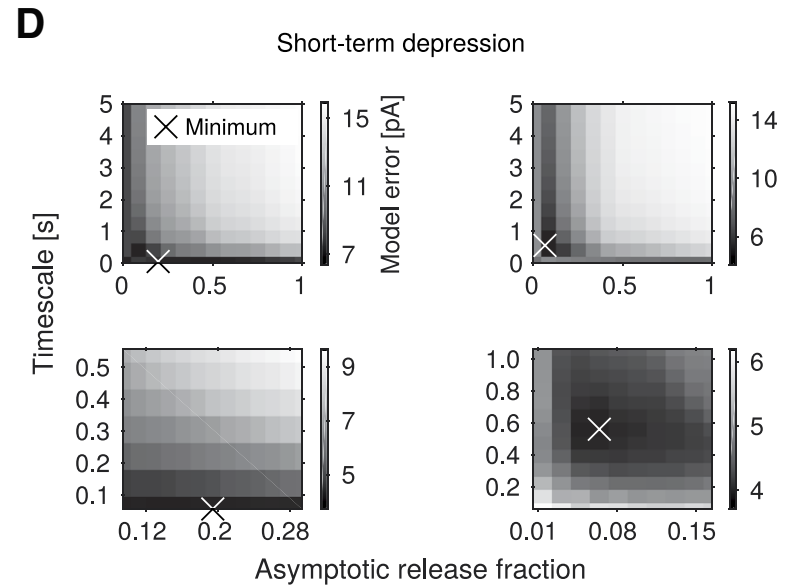

Figure 7. Modeling of a feedback inhibitory circuit. $\boldsymbol{A}$, Cell-averaged EPSPs measured in BCs (blue traces; control and epileptic animals, respectively, $n=5$ and $n=7$ ) and EPSPs obtained from the fit of the model to these data (orange traces). Data and fit for both the $50 \mathrm{~Hz}$ protocol (top) and the theta burst protocol (bottom) are shown. Scale bars hold for all EPSPs that are in the same row. $C$, Error between experimentally measured EPSPs and EPSPs obtained from the model as a function of the parameters describing short-term depression. Bottom, Close-up view of the error surface around the parameters obtained from the fit of the model to the data (crosses). $B, D$, Same as in $A, C$, but for the IPSCs measured in pyramidal cells in control and epileptic animals $(n=9$ and $n=7)$.

imum and to determine how sensitive the model error is to deviations from the found minimum, we computed the model error on a coarse-grained grid in parameter space. To visualize this approximate error surface, we projected the grid on the two key parameters describing short-term depression, asymptotic release fraction and timescale of replenishment, where all other parameters are optimized for a given combination of the two. The model only reproduces the data in control animals well if the asymptotic release fraction and the replenishment timescale lie close to the minimum found by the fit. In contrast, in the epileptic case, the model yields good results if either the asymptotic release fraction or the replenishment timescale is near minimal (Fig. 7C). This qualitative difference reflects the fact that short-term depression becomes negligible in epileptic animals (Figs. 1, 2; Table 4). Incorporation of synaptic facilitation did not markedly improve the fit in sham and epileptic animals, indicating that it is negligible in the mean population output (see also Fig. 3).

Next, we considered the BC-to-pyramidal cell synapse, again using a current-based model including STP. The already obtained model for the BC responses to excitatory stimulation allowed us to fit the model parameters describing the pyramidal cells and the BC-to-pyramidal cell synapse to the IPSC traces measured in response to stimulation in the alveus (Figs. 5, 6). Again, the fit to data obtained from both control animals and epileptic animals was good (Fig. $7 B$, top; $R^{2}=0.95$ and $R^{2}=0.93$ for control and epileptic animals, respectively). Using the same approach as before, we determined an approximate error surface over the parameters describing the short-term depression of the BC-to-pyramidal cell inhibitory synapse, which was not directly experimentally measured in this study (Fig. $7 D)$. The results revealed best fits for asymptotic release fractions of 0.064 versus 0.194 (shallow optimum) for epileptic and control animals, respectively (Table 5). This is consistent with experimental studies on BC output synapses in the pilocarpine model that have indicated a reduced release probability at BC synapses (Zhang and Buckmaster, 2009), perhaps due to changes in steps following vesicle docking, including priming, $\mathrm{Ca}^{2+}$ entry, $\mathrm{Ca}^{2+}$ coupling with exocytosis, or replenishment of the reserve pool (Buckmaster et al., 2016).

\section{Consequences of altered feedback circuits: altered burst transmission from CA3 via CA1}

We then asked what the consequences of the observed changes in the feedback circuit are for input-output conversion in the CA1 region. The experimental data show that recruitment of perisomatic inhibition in feedback networks is substantially altered, via both changes in intrinsic interneuron properties and their synaptic recruitment. These changes predict a substantial decrease in the initial inhibition that can be recruited when the CA1 pyramidal ensemble begins to discharge synchronously. Under conditions under which CA3 cells are synchronously active, as during epileptiform burst activity, 
Table 4. Parameter values found by the fitting procedure for the basket cell data in the control and epileptic case ${ }^{a}$

\begin{tabular}{|c|c|c|c|c|c|c|c|c|c|}
\hline & $\tau_{\mathrm{d}, \mathrm{BC}}(\mathrm{ms})$ & $\lambda$ & $\tau_{\mathrm{e}, \mathrm{BC}}(\mathrm{ms})$ & $\hat{l}_{\mathrm{e}, \mathrm{BC}}(\mathrm{mV})$ & $\tau_{\mathrm{RRP}, \mathrm{e}, \mathrm{BC}}(\mathrm{ms})$ & $u_{0, \mathrm{e}, \mathrm{BC}}$ & $\tau_{\mathrm{fac}, \mathrm{e}, \mathrm{BC}}(\mathrm{ms})$ & $u_{\mathrm{f}, \mathrm{e}, \mathrm{BC}}$ & Model error (mV) \\
\hline Search space & $10-100$ & $-1-0$ & $0.005-5$ & $0.02-20$ & $5-5000$ & $0.001-1$ & $5-5000$ & $0-1$ & - \\
\hline Sham-control & 55.5 & -0.952 & 1.790 & 6.82 & 841 & 0.185 & 5 & 0.000 & 0.212 \\
\hline Epileptic & 56.8 & -0.461 & 1.938 & 2.44 & 1856 & 0.018 & 5 & 0.000 & 0.219 \\
\hline
\end{tabular}

${ }^{a}$ The first row shows the parameter bounds within which the parameter space was searched for optimal values. $\tau_{\mathrm{d}, \mathrm{BC}}$, Membrane time constant; $\lambda$, strength of nonlinearity of membrane potential; $\tau_{\mathrm{e}, \mathrm{BC}}$, synaptic time constant; $\hat{l}_{\mathrm{l}, \mathrm{BC}}$, impact strength of released fraction of neurotransmitters; $\tau_{\mathrm{RRP}, \mathrm{e}, \mathrm{BC}}$, time constant of synaptic depression; $u_{0, \mathrm{e}, \mathrm{BC}}$, asymptotic release fraction; $\tau_{\mathrm{fac}, \mathrm{e}, \mathrm{BC}}$, time constant of synaptic facilitation; $u_{\mathrm{f}, \mathrm{e}, \mathrm{BC}}$, increase of release fraction after a presynaptic spike; Model error, error between data and model. Parameters correspond to the basket cell population or the pyramidal cell-to-basket cell synapse.

Table 5. Parameter values found by the fitting procedure for the pyramidal cell data in the control and epileptic case ${ }^{a}$

\begin{tabular}{|c|c|c|c|c|c|c|c|c|}
\hline & $\tau_{\mathrm{i}, \mathrm{PY}}(\mathrm{ms})$ & $\hat{l}_{\mathrm{i}, \mathrm{PY}}(\mathrm{pA})$ & $\tau_{\mathrm{RRP}, \mathrm{i}, \mathrm{PY}}(\mathrm{ms})$ & $u_{0, \mathrm{i}, \mathrm{PY}}$ & $\tau_{\mathrm{fac}, \mathrm{i}, \mathrm{PY}}(\mathrm{ms})$ & $u_{\mathrm{f}, \mathrm{i}, \mathrm{PY}}$ & $\hat{l}_{\mathrm{e}, \mathrm{BC}}(\mathrm{mV})$ & Model error (pA) \\
\hline Search space & $0.015-15$ & $0.1-500$ & $5-5000$ & $0.001-1$ & $5-5000$ & $0-1$ & $3-300$ & - \\
\hline Sham-control & 12.88 & 33.0 & 57 & 0.194 & 5 & 0.000 & 74.3 & 3.792 \\
\hline Epileptic & 9.66 & 30.2 & 561 & 0.064 & 5 & 0.000 & 33.5 & 3.711 \\
\hline
\end{tabular}

${ }^{a}$ The first row shows the parameter bounds within which we searched for the optimal values. $\tau_{\mathrm{i}, \mathrm{PY}}$, Synaptic time constant; $\hat{l}_{\mathrm{i}, \mathrm{PY}}$, impact strength of released fraction of neurotransmitters; $\tau_{\mathrm{RR}, \mathrm{i}, \mathrm{PY}}$, time constant of synaptic depression; $u_{0, \mathrm{i}, \mathrm{PY}}$ asymptotic release fraction; $\tau_{\mathrm{fac}, i, \mathrm{py}}$, time constant of synaptic facilitation; $u_{\mathrm{f}, \mathrm{i}, \mathrm{PY}}$, increase of release fraction after a presynaptic spike; Model error, error between data and model. Parameters correspond to the pyramidal cell population or the basket cell-to-pyramidal cell synapse.

feedback inhibition may then be particularly inefficient in controlling CA1 pyramidal cell excitability.

To test this prediction, we generated a model of the complete inhibitory feedback motif for the control and epileptic case, using the components described above (Fig. 8A; see Materials and Methods). The feedback circuit model was then probed with inputs from CA3 that were systematically varied. Specifically, the input was represented by a rate, which increased with various degrees of steepness, and then stayed constant for $80 \mathrm{~ms}$, thereafter dropping to zero again (Fig. $8 B ; r_{\mathrm{IN}}$, example for rate increase from 0 to $70 \mathrm{~Hz}$ over $150 \mathrm{~ms}$ ). These parameters were chosen based on the frequency and duration of CA3 ripples or epileptic bursts (Foffani et al., 2007; Jiruska et al., 2010; Oliva et al., 2018). The entirety of the epilepsy-induced changes led to decreased $\mathrm{BC}$ activity and increased pyramidal cell activity (Fig. $8 B ; r_{\mathrm{BC}}$ and $\mathrm{r}_{\mathrm{PY}}$, respectively; Fig. $\left.8 C, D\right)$. These changes were robust over a wide range of input rise times and maximal rates (Fig. 8C,D). Notably, the increase of pyramidal cell activity was especially pronounced for short rise times, which are typical for the initial phase of epileptic bursts (Fig. 8D, right). Hence, these results predict that the changes in CA1 during development of epilepsy promote the transmission of epileptic bursts from CA3 to other parts of the brain.

\section{Discussion}

In this paper, we demonstrate and quantify major functional changes in feedback inhibition in chronic epilepsy, consisting of both intrinsic and synaptic changes. Collectively, these changes result in altered dynamics of CA1 feedback inhibition and predict decreased filtering of burst-like activity from the CA3 region.

Changed dynamics of feedback inhibition in chronic epilepsy Feedback inhibition plays a crucial role in controlling excitability of pyramidal neurons. Feedback excitation of perisomatically inhibiting interneurons normally shows strong short-term depression, as shown previously (Pouille and Scanziani, 2004; Müller et al., 2012; Pothmann et al., 2014). This is markedly changed in epilepsy, leading to a profound reduction of initial perisomatic inhibition. To assess the consequences of these and the other observed changes on the input-output properties of the CA1 region, we developed a simple, biologically plausible CA1 circuit model of the feedback inhibitory motif. The model was systematically fitted to our experimental data generated for the different elements in the feedback circuit. Probing it with inputs from CA3, we find that the epilepsy-associated changes in the feedback inhibitory motif cause an increased CA1 output. Notably, the increase is particularly pronounced in the case of steep rises of the input signal from CA3, which are typical for the initial phase of epileptic bursts. This indicates that the changes in CA1 during development of epilepsy foster the transmission of epileptic bursts from CA3 to other parts of the brain.

These findings may also be relevant to burst-like inputs occurring during sharp-wave ripple oscillations, which are driven by CA3 pyramidal neurons. In the normal brain, both basket and PD interneurons are efficiently recruited by such activity patterns. Accordingly, feedback inhibition impinging on the soma and proximal dendrites during these input patterns is strong. In epileptic animals, sharp-wave ripples are also observed, with distinct and more variable spectral features (Ibarz et al., 2010; Valero et al., 2017). In keeping with the predictions of our model, CA1 cells fired more in epileptic animals during sharp-wave ripples, as well as participating indiscriminately in multiple types of sharp-wave ripple events (Valero et al., 2017). Moreover, these changes seemed to be due to altered excitation-inhibition balance, according to intracellular measurements of synaptic conductances, as well as pharmacological experiments. Thus, the circuit abnormality we have demonstrated appears to be relevant in vivo. Moreover, the deficit in inhibitory efficacy and timing of inhibition may interfere with proper ensemble selection of CA1 ensembles during sharp-wave ripples, which would be expected to degrade the information capacity of CA1 networks (Valero et al., 2017). Because precise activation of CA1 ensembles during sharp-wave ripples is important for memory formation (Girardeau et al., 2009; Buzsáki, 2015), these findings are likely also relevant for impaired memory formation in the epileptic hippocampus.

What is the mechanism underlying changes in the dynamics of feedback inhibition? At feedback excitatory synapses targeting BCs, a significant reduction in paired pulse facilitation was observed, consistent with a reduction in release probability. An interesting candidate mechanism controlling release probability at excitatory synapses onto OLM interneurons versus BCs is driven by the differential expression of the transmembrane signaling molecule Elfn1 (Sylwestrak and Ghosh, 2012). Interestingly, epilepsy-associated mutations of this gene were found in patients suffering from absence epilepsy and juvenile myoclonic epilepsy (Tomioka et al., 2014).

In addition to the changes in short-term dynamics, we describe a strongly decreased input resistance of BCs and OLM neurons that results in a pronounced decrease in intrinsic excit- 
A
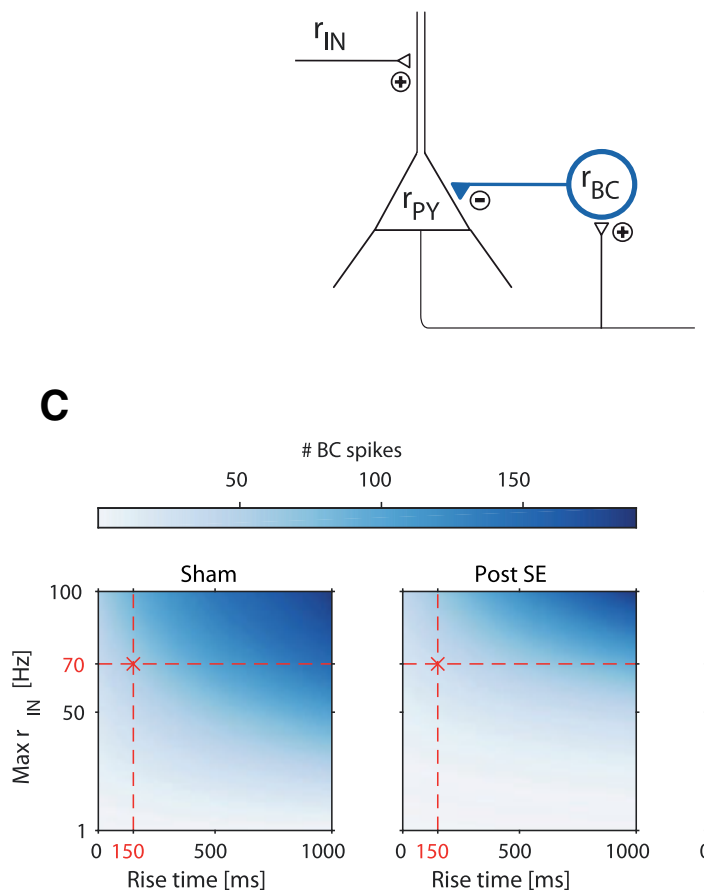

D

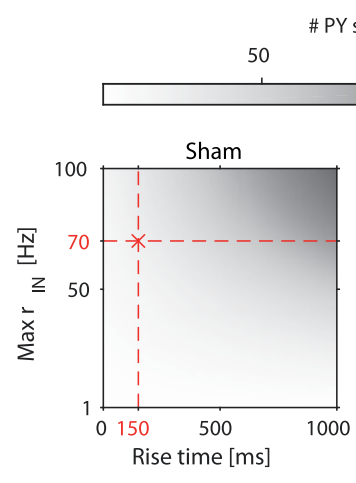

PY spikes

100

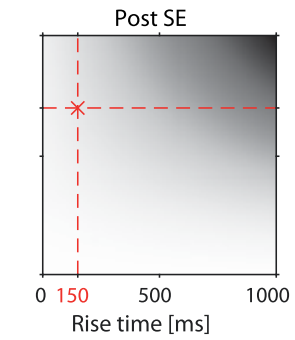

\# BC spikes

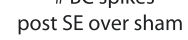

0.2

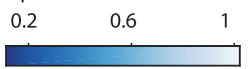

Post SE
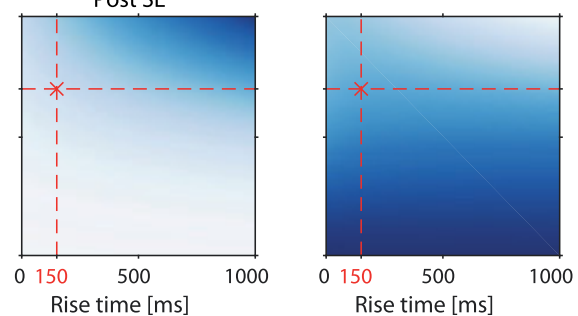

\# PY spikes post SE over sham

$\begin{array}{lll}1.2 & 1.4 & 1.6\end{array}$
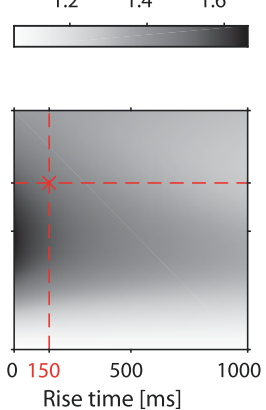

B
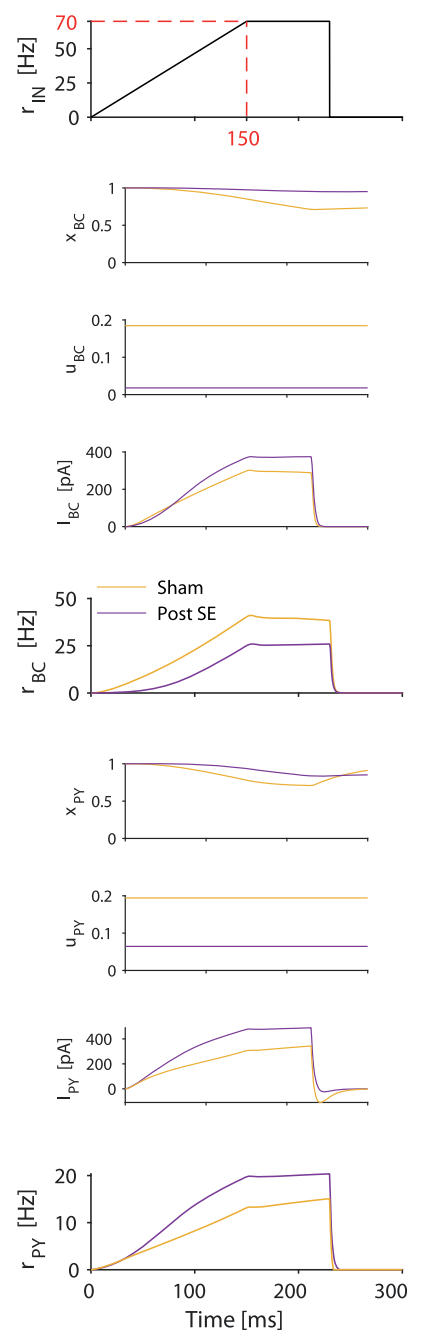

Figure 8. Increased burst transmission caused by altered dynamics of feedback inhibition. $\boldsymbol{A}$, Schematic diagram of the model components. $\boldsymbol{B}$, Example time evolution of all model variables in the control (orange) and epileptic case (purple) for an example input (top). $x_{\mathrm{BC}}$ is the fraction of available neurotransmitter in the readily releasable pool of vesicles, and $u_{\mathrm{BC}}$ is the release fraction of neurotransmitter for the pyramidal cell-to-BC synapse. $I_{B C}$ is the total input current to the $B C$. $x_{\mathrm{py}}, u_{\mathrm{py}}$, and $I_{\mathrm{py}}$ are the corresponding quantities for the $B C$-to-pyramidal cell synapse and the pyramidal cells (see Materials and Methods). $C$, Number of spikes of the BCs in the epileptic case and in the control case (left subpanels) and their ratio for different input rise times and maximal firing rates (right subpanel). Red lines indicate the parameters shown in $\boldsymbol{B}$. D, Same as in $\boldsymbol{C}$, but for pyramidal cells.

ability. Notably, in contrast to the synaptic mechanisms described above, these changes will impact interneuron excitability regardless of whether they are being recruited in feedback or feedforward circuits.

In the pilocarpine model, a number of studies have addressed the dysfunction of GABAergic systems. Consistent with our results regarding GABAergic bouton density, the number of GABAergic boutons innervating CA1 pyramidal cell somata was not reduced in the pilocarpine model (Wyeth et al., 2010) as well as human tissue (Wittner et al., 2005). There was also no loss of spontaneous GABAergic IPSCs in CA1 pyramidal neurons (Williams et al., 1993; Esclapez et al., 1997; Cossart et al., 2001). However, there are more subtle effects regarding changes in specific subtypes of perisomatic interneurons in this model. There is a selective reduction in perisomatic CA1 pyramidal cell innervation from CCK-expressing BCs, with a loss of
CCK and CB1 receptor-expressing boutons, whereas PV-expressing boutons (Wyeth et al., 2010) and PV-expressing somata in the pyramidal cell layer (André et al., 2001) were unchanged. We did not differentiate between PV and CCK BCs in our analyses, but our finding of altered dynamics of feedback excitation may compound changes due to an altered composition of perisomatic inhibition.

In addition, changes in proximal dendritic inhibition have been described. For instance, detailed analysis of interneurons in the pilocarpine model of epilepsy shows that there is profound loss of PV neurons in the SO of CA1, with loss of PV-immunoreactive axon terminals in stratum oriens, suggesting that PVimmunoreactive dendrite targeting interneuron subtypes may be reduced (André et al., 2001).

Finally, consistent with our results from OLM neurons, somatostatin-immunoreactive neurons in stratum oriens were shown to be conserved in the pilocarpine model of epilepsy, both 
regarding their cell numbers and their projections in CA1 (Cossart et al., 2001; Peng et al., 2013; but see Houser and Esclapez, 1996). However, two factors may conspire to reduce dendritic inhibition. First, a reduction in release probability at inhibitory synapses targeting the apical dendrites has been described (Cossart et al., 2001). Second, we have described a marked reduction in input resistance of OLM neurons and rheobase, implying markedly reduced excitability. Accordingly, even though feedback excitation of OLM neurons is unaltered, their capability to provide dendritic CA1 inhibition is likely to be severely impaired. Thus, numerous changes may conspire to cripple both dendritic and somatic GABAergic function.

The changes we describe interact with changes in excitatory neurons. One prominent change in the CA1 region is an increase in intrinsic bursting behavior (Su et al., 2002; Yaari et al., 2007; Becker et al., 2008). This change is likely relevant for seizure initiation because spontaneously occurring burst discharges precede seizure-like activity in hippocampal slices (Su et al., 2002). Our results predict that synchronized bursting of pyramidal neurons would be less controlled by recurrent inhibition. This would hold true if the bursting is driven by rapid increases of CA3 activity.

In conclusion, we demonstrate multiple changes in interneurons involved in feedback inhibition and in their recruitment. These conspire to cause pronounced impairment of a canonical inhibitory motif in chronic epilepsy, which may be an important contributor to the generation and spread of aberrant activity.

\section{References}

André V, Marescaux C, Nehlig A, Fritschy JM (2001) Alterations of hippocampal GABAergic system contribute to development of spontaneous recurrent seizures in the rat lithium-pilocarpine model of temporal lobe epilepsy. Hippocampus 11:452-468.

Ang CW, Carlson GC, Coulter DA (2005) Hippocampal CA1 circuitry dynamically gates direct cortical inputs preferentially at theta frequencies. J Neurosci 25:9567-9580.

Becker AJ, Pitsch J, Sochivko D, Opitz T, Staniek M, Chen CC, Campbell KP, Schoch S, Yaari Y, Beck H (2008) Transcriptional upregulation of Cav3.2 mediates epileptogenesis in the pilocarpine model of epilepsy. J Neurosci 28:13341-13353.

Buckmaster PS, Yamawaki R, Thind K (2016) More docked vesicles and larger active zones at basket cell-to-granule cell synapses in a rat model of temporal lobe epilepsy. J Neurosci 36:3295-3308.

Buzsáki G (2015) Hippocampal sharp wave-ripple: a cognitive biomarker for episodic memory and planning. Hippocampus 25:1073-1188.

Cossart R, Dinocourt C, Hirsch JC, De Felipe J, Esclapez M, Bernard C, Merchan-Perez A (2001) Dendritic but not somatic GABAergic inhibition is decreased in experimental epilepsy. Nat Neurosci 4:52-62.

Esclapez M, Hirsch JC, Khazipov R, Ben-Ari Y, Bernard C (1997) Operative GABAergic inhibition in hippocampal CA1 pyramidal neurons in experimental epilepsy. Proc Natl Acad Sci U S A 94:12151-12156.

Foffani G, Uzcategui YG, Gal B, Menendez de la Prida L (2007) Reduced spike-timing reliability correlates with the emergence of fast ripples in the rat epileptic hippocampus. Neuron 55:930-941.

Freund TF, Buzsáki G (1996) Interneurons of the hippocampus. Hippocampus 6:347-470.

Girardeau G, Benchenane K, Wiener SI, Buzsáki G, Zugaro MB (2009) Selective suppression of hippocampal ripples impairs spatial memory. Nat Neurosci 12:1222-1223.

Houser CR, Esclapez M (1996) Vulnerability and plasticity of the GABA system in the pilocarpine model of spontaneous recurrent seizures. Epilepsy Res 26:207-218.

Ibarz JM, Foffani G, Cid E, Inostroza M, Menendez de la Prida L (2010) Emergent dynamics of fast ripples in the epileptic hippocampus. J Neurosci 30:16249-16261.
Jiruska P, Finnerty GT, Powell AD, Lofti N, Cmejla R, Jefferys JG (2010) Epileptic high-frequency network activity in a model of non-lesional temporal lobe epilepsy. Brain 133:1380-1390.

Longair MH, Baker DA, Armstrong JD (2011) Simple neurite tracer: open source software for reconstruction, visualization and analysis of neuronal processes. Bioinformatics 27:2453-2454.

Markram H, Wang Y, Tsodyks M (1998) Differential signaling via the same axon of neocortical pyramidal neurons. Proc Natl Acad Sci U S A 95:5323-5328.

Miles R (1990) Synaptic excitation of inhibitory cells by single CA3 hippocampal pyramidal cells of the guinea-pig in vitro. J Physiol 428:61-77.

Müller C, Beck H, Coulter D, Remy S (2012) Inhibitory control of linear and supralinear dendritic excitation in CAl pyramidal neurons. Neuron 75:851-864.

Oliva A, Fernández-Ruiz A, Fermino de Oliveira E, Buzsáki G (2018) Origin of gamma frequency power during hippocampal sharp-wave ripples. Cell Rep 25:1693-1700.e4.

Peng Z, Zhang N, Wei W, Huang CS, Cetina Y, Otis TS, Houser CR (2013) A reorganized GABAergic circuit in a model of epilepsy: evidence from optogenetic labeling and stimulation of somatostatin interneurons. J Neurosci 33:14392-14405.

Pothmann L, Müller C, Averkin RG, Bellistri E, Miklitz C, Uebachs M, Remy S, Menendez de la Prida L, Beck H (2014) Function of inhibitory micronetworks is spared by $\mathrm{Na}^{+}$channel-acting anticonvulsant drugs. J Neurosci 34:9720-9735.

Pouille F, Scanziani M (2004) Routing of spike series by dynamic circuits in the hippocampus. Nature 429:717-723.

Smith MA, Ellis-Davies GC, Magee JC (2003) Mechanism of the distancedependent scaling of Schaffer collateral synapses in rat CA1 pyramidal neurons. J Physiol 548:245-258.

Somogyi P, Klausberger T (2005) Defined types of cortical interneurone structure space and spike timing in the hippocampus. J Physiol 562:9-26.

Spruston N, Jonas P, Sakmann B (1995) Dendritic glutamate receptor channels in rat hippocampal CA3 and CA1 pyramidal neurons. J Physiol 482:325-352.

Su H, Sochivko D, Becker A, Chen J, Jiang Y, Yaari Y, Beck H (2002) Upregulation of a T-type $\mathrm{Ca}^{2+}$ channel causes a long-lasting modification of neuronal firing mode after status epilepticus. J Neurosci 22:3645-3655.

Sylwestrak EL, Ghosh A (2012) Elfn1 regulates target-specific release probability at CA1-interneuron synapses. Science 338:536-540.

Tomioka NH, Yasuda H, Miyamoto H, Hatayama M, Morimura N, Matsumoto Y, Suzuki T, Odagawa M, Odaka YS, Iwayama Y, Won Um J, Ko J, Inoue Y, Kaneko S, Hirose S, Yamada K, Yoshikawa T, Yamakawa K, Aruga J (2014) Elfn1 recruits presynaptic mGluR7 in trans and its loss results in seizures. Nat Commun 5:4501.

Valero M, Averkin RG, Fernandez-Lamo I, Aguilar J, Lopez-Pigozzi D, Brotons-Mas JR, Cid E, Tamas G, Menendez de la Prida L (2017) Mechanisms for selective single-cell reactivation during offline sharpwave ripples and their distortion by fast ripples. Neuron 94:12341247.e7.

Williams S, Vachon P, Lacaille JC (1993) Monosynaptic GABA-mediated inhibitory postsynaptic potentials in cal pyramidal cells of hyperexcitable hippocampal slices from kainic acid-treated rats. Neuroscience 52:541554.

Wittner L, Eross L, Czirják S, Halász P, Freund TF, Maglóczky Z (2005) Surviving CAl pyramidal cells receive intact perisomatic inhibitory input in the human epileptic hippocampus. Brain 128:138-152.

Wyeth MS, Zhang N, Mody I, Houser CR (2010) Selective reduction of cholecystokinin-positive basket cell innervation in a model of temporal lobe epilepsy. J Neurosci 30:8993-9006.

Yaari Y, Yue C, Su H (2007) Recruitment of apical dendritic T-type $\mathrm{Ca}^{2+}$ channels by backpropagating spikes underlies de novo intrinsic bursting in hippocampal epileptogenesis. J Physiol 580:435-450.

Ylinen A, Soltész I, Bragin A, Penttonen M, Sik A, Buzsáki G (1995) Intracellular correlates of hippocampal theta rhythm in identified pyramidal cells, granule cells, and basket cells. Hippocampus 5:78-90.

Zhang W, Buckmaster PS (2009) Dysfunction of the dentate basket cell circuit in a rat model of temporal lobe epilepsy. J Neurosci 29:78467856. 